\title{
Patrolling monocytes promote the pathogenesis of early lupus-like glomerulonephritis
}

\author{
Jeeba Kuriakose, ${ }^{1}$ Vanessa Redecke, ${ }^{1}$ Cliff Guy, ${ }^{2}$ Jingran Zhou, ${ }^{1}$ Ruiqiong Wu, ${ }^{1}$ Sirish K. Ippagunta, ${ }^{1}$ Heather Tillman, ${ }^{3}$ \\ Patrick D. Walker, ${ }^{4}$ Peter Vogel, ${ }^{3}$ and Hans Häcker ${ }^{1}$ \\ 'Department of Infectious Diseases, ${ }^{2}$ Department of Immunology, and ${ }^{3}$ Department of Pathology, St. Jude Children's Research Hospital, Memphis, Tennessee, USA. ${ }^{4}$ Arkana Laboratories, Little Rock, Arkansas, USA.
}

\begin{abstract}
Systemic lupus erythematosus (SLE) is a complex autoimmune disease with genetic and environmental contributions. Hallmarks of the disease are the appearance of immune complexes (IC) containing autoreactive Abs and TLR-activating nucleic acids, whose deposition in kidney glomeruli is suspected to promote tissue injury and glomerulonephritis (GN). Here, using a mouse model based on the human SLE susceptibility locus TNFAIP3-interacting protein 1 (TNIP1, also known as ABIN1), we investigated the pathogenesis of GN. We found that GN was driven by TLRs but, remarkably, proceeded independently of ICs. Rather, disease in 3 different mouse models and patients with SLE was characterized by glomerular accumulation of patrolling monocytes (PMos), a cell type with an emerging key function in vascular inflammation. Consistent with such function in CN, monocyte-specific deletion of ABIN1 promoted kidney disease, whereas selective elimination of PMos provided protection. In contrast to GN, PMo elimination did not protect from reduced survival or disease symptoms such as IC generation and splenomegaly, suggesting that GN and other inflammatory processes are governed by distinct pathogenic mechanisms. These data identify TLR-activated PMos as the principal component of an intravascular process that contributes to glomerular inflammation and kidney injury.
\end{abstract}

\section{Introduction}

Forty to seventy percent of all patients with systemic lupus erythematosus (SLE) eventually develop overt nephritis, and despite treatment, $10 \%-30 \%$ of these patients progress to end-stage kidney disease requiring dialysis or kidney transplantation (1). Lupus nephritis is considered an immune complex-mediated (ICmediated) form of glomerulonephritis (GN) caused by characteristic glomerular deposition of Igs and complement components. Autoreactive Abs in these complexes are, at least in part, directed against nuclear antigens, including dsDNA, whose appearance in the serum of patients with lupus represents a diagnostic criterion for SLE in common classifications (2). Although the pathogenic relevance of ICs in lupus nephritis remains to be formally tested, glomerular IC deposition and consequent complement fixation are thought to provide the basis for complement-mediated cytotoxicity and end-organ damage. ICs can also activate nucleic acid-recognizing TLRs, i.e., TLR7, TLR8, and TLR9, on immune cells including $\mathrm{B}$ cells, providing a possible explanation for the perpetuation of inflammation (3-6). In lupus, the function of TLRs, particularly those recognizing nucleic acids, is supported by a significant body of evidenceincluding (a) data from human patients showing nucleic acid-containing complexes in the serum of patients with SLE (46); (b) data showing the therapeutic efficacy of chloroquine, which prevents TLR activation by nucleic acids (7); (c) data from mouse

Authorship note: JK and VR contributed equally to this work. JZ is deceased. Conflict of interest: The authors have declared that no conflict of interest exists. Copyright: () 2019, American Society for Clinical Investigation.

Submitted: September 25, 2018; Accepted: March 12, 2019; Published: April 29, 2019. Reference information: J Clin Invest. 2019;129(6):2251-2265

https://doi.org/10.1172/JCI125116 models, in which constitutive TLR activity by increased gene expression drives inflammatory disease $(8,9)$; and, conversely, (d) data showing that genetic deletion of MyD88 or combined deletion of TLR7 and TLR9 ameliorates disease (10-13). Together, these observations support a model in which autoimmunity in the form of B cell-derived autoreactive Abs causes GN. Contrary to this simple scenario, B cell-directed therapies have not produced the expected therapeutic efficacy and have not been approved by the US FDA to treat lupus nephritis (14). Thus, it seems possible that the current pathogenic concept of lupus nephritis may be incomplete and that other B cell-independent mechanisms exist. This idea is supported by the more than 50 genetic SLE susceptibility loci identified so far, whose known (or predicted) gene products have well-established functions outside the adaptive immune system $(15,16)$. One characterized susceptibility locus for SLE and lupus nephritis is TNIP1 (TNFAIP3-interacting protein 1; also referred to as $A B I N 1$, A20-binding inhibitor of NF- $\kappa$ B activation 1), which was identified by GWAS (17-19). Patients with TNIP1 SNPs demonstrated reduced mRNA and protein expression, indicating a hypomorphic (loss-of-function) phenotype (20). ABIN1 has been characterized as a negative regulator of the TNFR, TLR, and IL-17R pathways, and, consistent with its genetic link to human SLE, ABIN1-deficient mice develop a systemic lupus-like disease that includes major characteristics of human SLE, including the generation of autoreactive Abs and severe GN (21-23). In vitrogenerated DCs and macrophages were shown to exhibit TLRmediated gene deregulation upon loss of ABIN1 function (22). The contribution of other cell types to immune pathology, including patrolling monocytes (PMos), one of the two monocyte subtypes defined in the peripheral blood ( $\mathrm{PB})$, has not been investigated to our knowledge. Independent work demonstrated the sensitivity 
A
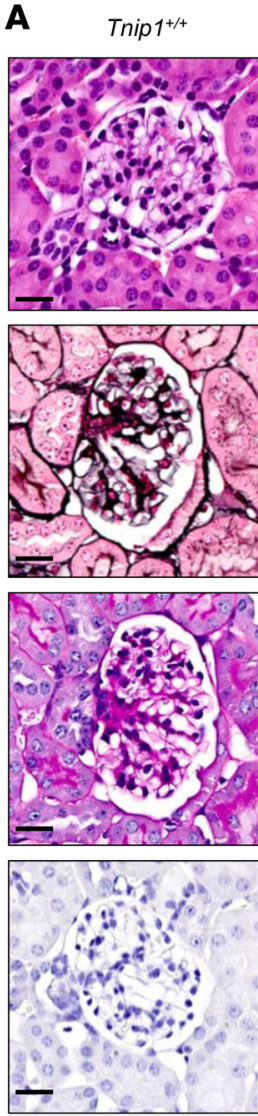

Tnip 1--
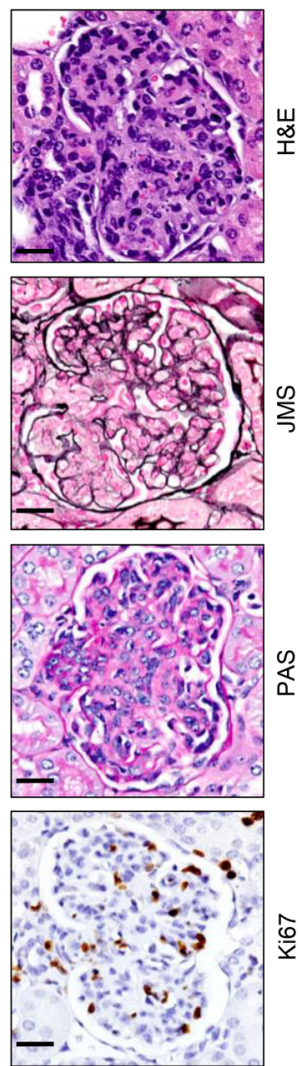

B

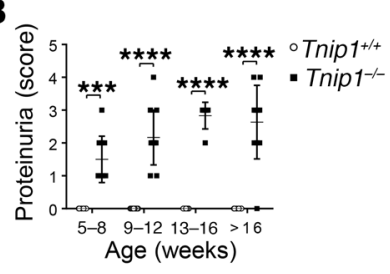

C

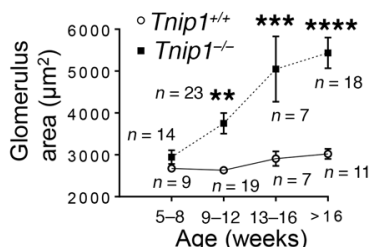

D
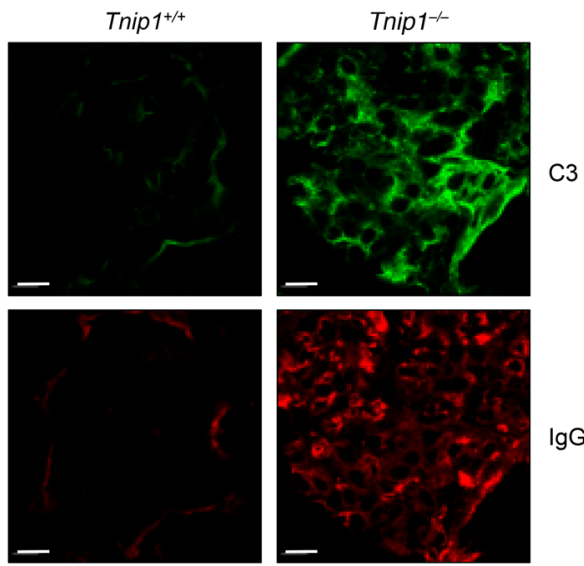

E

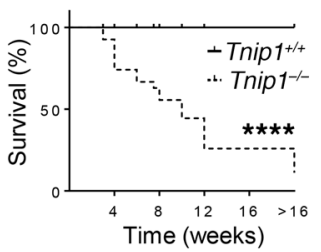

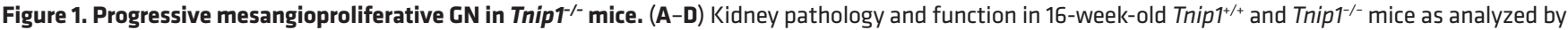
(A) histology based on H\&E, JMS, and PAS staining and staining for Abs against Ki67, (B) measurement of protein concentration in urine, (C) measurement of average glomerulus size, and (D) IF of kidney using Abs against C3 and IgG. Representative images of glomeruli are shown (scale bars: $20 \mu \mathrm{m}$ ). (E) Survival of Tnip $1^{+/+}$and Tnip1 $1^{-/-}$mice. Data represent the mean \pm SEM (B) or \pm SD (C). ${ }^{* *} P<0.01$, ${ }^{* * *} P<0.001$, and ${ }^{* * * *} P<0.0001$, by 2-way ANOVA with Sidak's multiple comparisons test (B and $\mathbf{C}$ ) and Mantel-Cox test (E). Symbols represent data from individual mice.

of PMos for TLR-activating nucleic acids, which control PMo biology on different levels, including cell maturation and chemokine expression $(24,25)$. PMos have received particular attention more recently, given their emerging, specialized function in the blood vasculature during inflammatory insults (26). It is apparent from these studies that endothelial tissue injury and inflammatory triggers, experimentally inflicted by cytotoxic Abs or by TLR activation, lead to the swift recruitment of PMos, which in turn orchestrate subsequent phases of the ensuing inflammatory response, including the attraction of neutrophils that mediate endothelial injury (26-28). Increased numbers of PMos were also detected in the PB and kidney glomeruli of patients with SLE, indicating a possible role in lupus nephritis $(25,29,30)$. Here, we characterize GN in ABIN1-deficient mice and 2 additional independent lupus models, identifying PMos as a principal cell type mediating tissue injury in lupus-like nephritis.

\section{Results}

ABIN1-deficient mice develop mesangioproliferative GN. Proliferative forms of lupus nephritis carry a high risk of progression to end-stage renal disease (ESRD) and are characterized by glomerular hypercellularity and matrix accumulation with Ig and complement deposits (31). Histological examination of H\&E-stained kidney tissue from ABIN1-deficient mice revealed diffuse global hypercellularity and matrix accumulation, with multifocal endocapillary proliferation and scattered pyknotic nuclei (Figure 1A). Disease progressed over time, as assessed by proteinuria and the increasing size of the affected glomeruli of ABIN1-deficient mice, a quantitative measure that correlated well with the histopathological grade and severity of nephritis in the mice (Figure 1, B and C, and Supplemental Figure 1, A-C; supplemental material available online with this article; https://doi.org/10.1172/ JCI125116DS1) (32). Jones methenamine silver (JMS) and periodic acid-Schiff (PAS) staining revealed global and segmental endocapillary proliferation and increased mesangial matrix, whereas Ki67-staining highlighted the markedly increased number of proliferating cells in glomeruli (Figure 1A). Although diseased glomeruli also contained significantly increased numbers of $\mathrm{CD} 45^{+}$leukocytes, the majority of $\mathrm{Ki} 67^{+}$cells were identified as DESMIN $^{+}$mesangial cells (Supplemental Figure 1, D and E). Consistent with an IC-mediated form of GN, the glomeruli of ABIN1-deficient mice showed strong staining for $\operatorname{IgG}$ and C3 (Figure 1D). Older mice developed more severe glomerular changes, including capsular adhesions and obliteration of the glomerular architecture (Supplemental Figure 1, B and C). Increased GN severity was paralleled by lethality (Figure 1E), however, the 
A

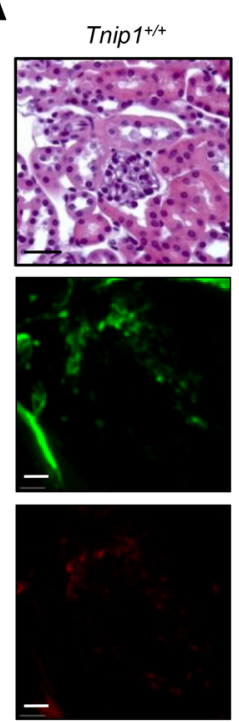

B

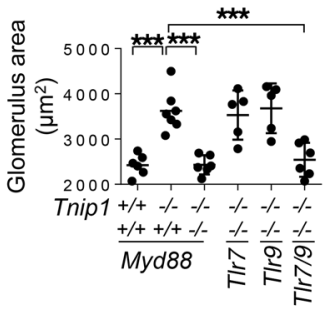

$\operatorname{Tnip1}^{-1}$
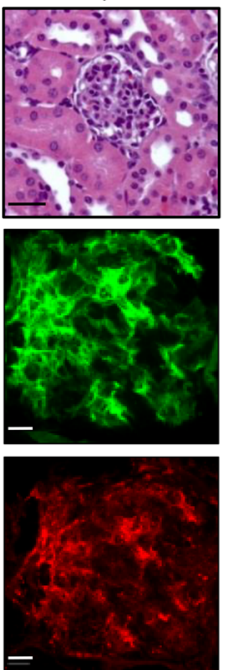

C 응
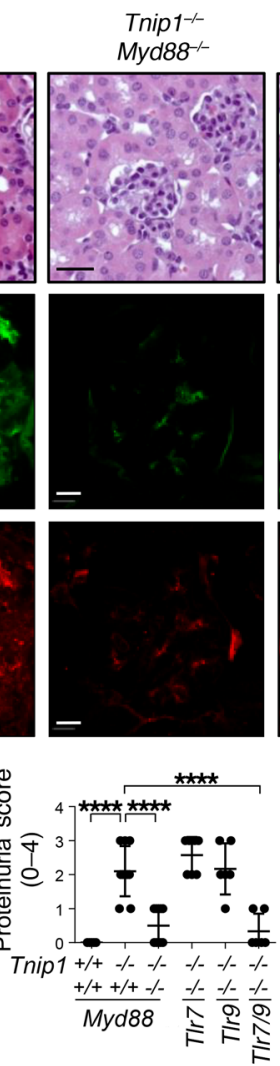
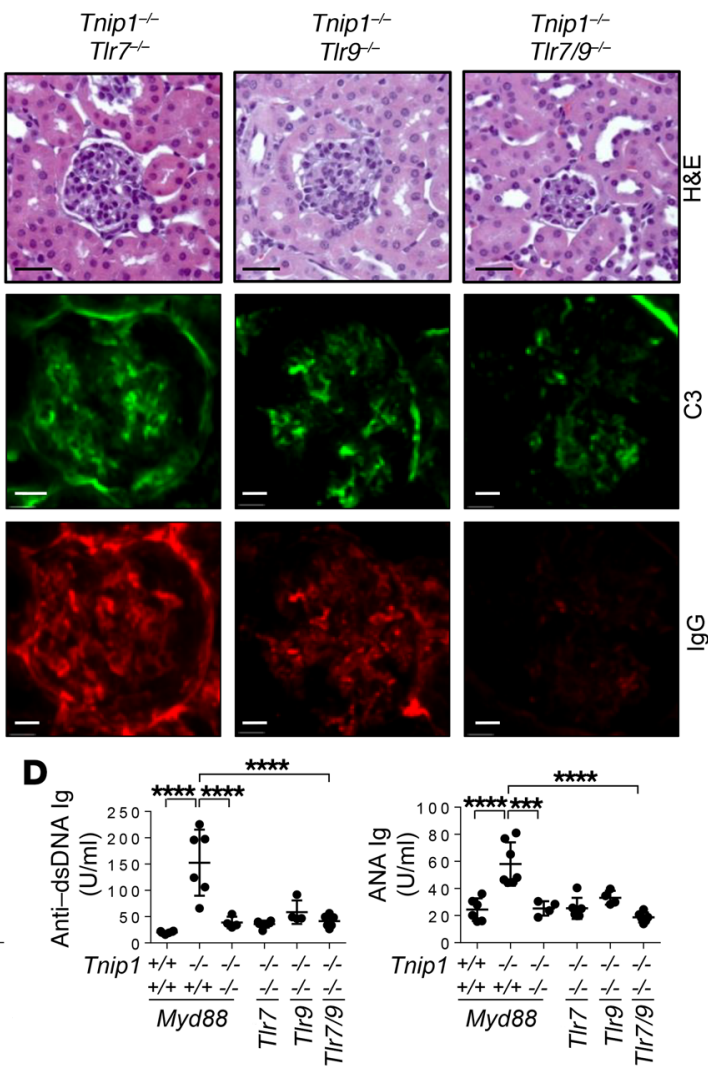

Figure 2. Signaling of nucleic acid-recognizing TLRs promotes mesangioproliferative GN. (A-C) Kidney pathology and function of 16-week-old mice of the indicated genotypes as analyzed by (A) histology based on H\&E staining and staining for Abs against C3 and IgG, (B) measurement of average glomerulus size, and (C) measurement of protein concentration in urine. Representative images of glomeruli are shown (scale bars: $20 \mu \mathrm{m})$. (D) Levels of anti-dsDNA Ig and ANA Ig in serum from 16-week-old mice of the indicated genotypes. Data represent the mean \pm SD. ${ }^{* * *} P<0.001$ and ${ }^{* * * *} P<0.0001$ by 1-way ANOVA with Tukey's multiple comparisons test. Symbols represent data from individual mice.

residual glomerular filtration rate (GFR) of approximately $40 \%$ to $70 \%$ suggested that kidney injury was probably not the sole cause of death (Supplemental Figure 1F).

Collectively, these parameters confirm the diagnosis of a progressive, mesangioproliferative form of GN accompanied by IC deposits, following a disease process akin to proliferative forms of human lupus nephritis. Given the experimental constraint of increased lethality of the older mice, we focused on 12to 16-week-old mice, in which the GN pathology was significant and could be quantified by proteinuria and glomerular size.

Lupus nephritis is mediated via nucleic acid-recognizing TLRs. As mentioned above, different lines of evidence indicate an important role of TLRs in lupus, particularly TLR7 and TLR9, which recognize nucleic acids $(3-6,10,13)$. Thus, we tested the role of TLR7 and TLR9 in nephritis development in ABIN1-deficient mice. In these studies, we included MyD88, the common signaling molecule of TLR7 and TLR9, which we had also used as bait to identify ABIN1 as part of the TLR signaling complex $(22,33,34)$. Consistent with a major role of MyD88-dependent signal transduction in lupus nephritis, we found that Tnip1 ${ }^{--} \mathrm{Myd} 88^{---}$mice were protected from inflammatory disease symptoms including GN, proteinuria, formation of autoreactive Abs, and splenomegaly, as well as from early lethality (Figure 2, A-D, and Supplemental Figure 2, $\mathrm{A}$ and $\mathrm{B})$.
Neither deletion of TLR7 nor TLR9 alone produced significant benefits for GN, although splenomegaly was ameliorated (for TLR7), and anti-dsDNA Ig and antinuclear Ab Ig (ANA Ig) levels in the serum were reduced (for TLR7 and TLR9) (Figure 2, A-D, and Supplemental Figure 2, A and B). Importantly, combined deletion of both receptors led improved survival and a marked reduction of disease symptoms including kidney pathology, proteinuria, serum levels of anti-dsDNA and ANA Ig, glomerular IC deposits, and splenomegaly (Figure 2, A-D, and Supplemental Figure 2, A and B). We note that the adverse effect of deletion of TLR9 alone observed in some lupus models, most likely mediated by (unexplained) upregulation of pathogenic TLR7, was not observed in Tnip1 ${ }^{-/}$mice $(35,36)$. Still, from a therapeutic standpoint, data obtained from various mouse models suggest that interference with both nucleic acid-recognizing receptors is the most efficacious.

GN proceeds independently of lymphocytes, autoreactive Abs, and C3. Using fetal liver-chimeric mice, we previously showed that lupus-like disease in ABIN1-deficient mice is mediated by hematopoietic cells (22). To investigate the function of lymphocytes and the impact of B cell-derived Abs, we crossed Tnip1 ${ }^{-/}$mice with Rag1-deficient mice, which lack mature $\mathrm{T}$ and $\mathrm{B}$ cells (37). Interestingly, despite the absence of Ig deposits, all pathological criteria characterizing GN, as well as systemic disease parameters, were maintained in the nnip1 $^{-/-}$Rag1 $^{-/}$mice, including glomerular C3 
A
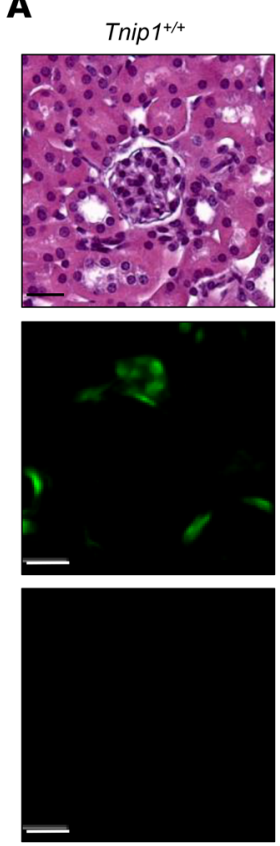

B

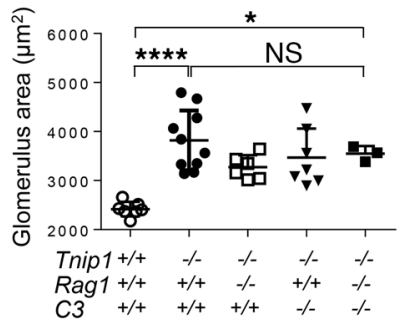

Tnip1-1- $^{-1}$
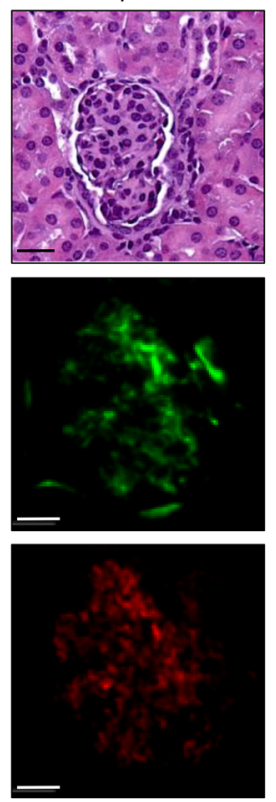

C
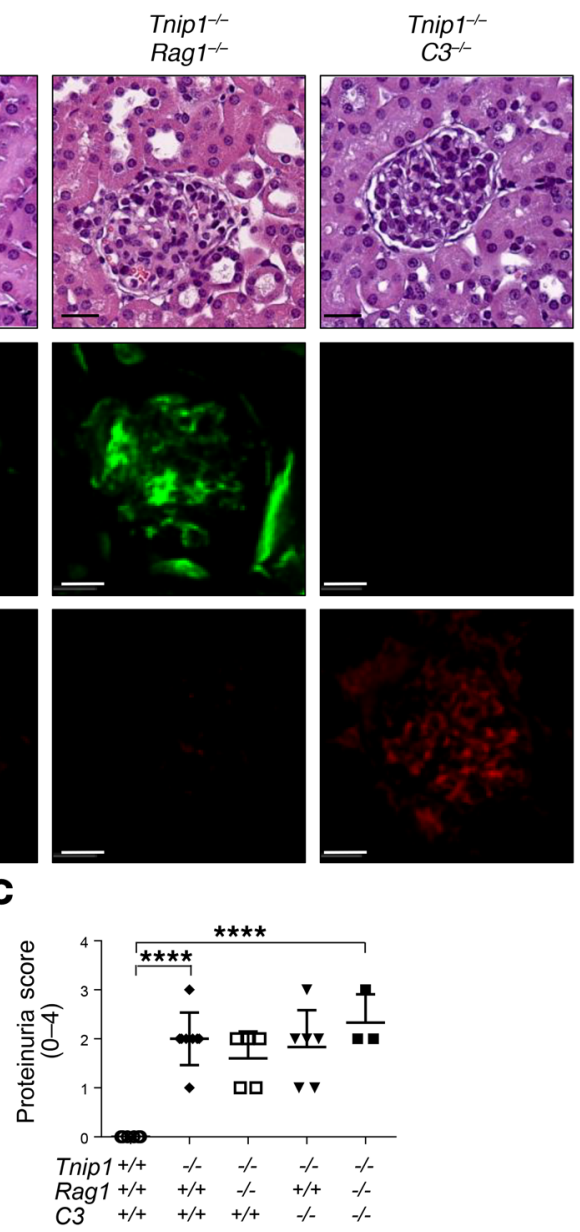

Figure 3. Mesangioproliferative phase of GN proceeds independently of glomerular IC and C3. (A-C) Kidney pathology and function of 12-week-old mice of the indicated genotypes as analyzed by (A) histology based on H\&E staining and staining for Abs against C3 and IgG, (B) measurement of average glomerulus size, and (C) measurement of protein concentration in urine. Representative images of glomeruli are shown (scale bars: $20 \mu \mathrm{m}$ ). Symbols represent data from individual mice. Data represent the mean $\pm \mathrm{SD}$. ${ }^{*} P<0.05$ and ${ }^{* * *} P<0.0001$, by 1-way ANOVA with Tukey's multiple comparisons test.

deposits, increased glomerular size, proteinuria, reduced survival, and splenomegaly (Figure 3, A-C, and Supplemental Figure 2, C and D). We note that similar results related to IC-independent GN development were also obtained in lupus-prone MRL/lpr mice, in which Ig secretion was genetically prevented (38). Given the deposition of C3 in the glomeruli of Tnip1 $1^{-/-}$Rag1 $^{-/-}$mice in the absence of Ig and a possible function of the alternative complement pathway, we crossed ABIN1-deficient mice with mice deficient for $C 3$, the common component of classic and alternative complement pathways (39). Still, neither deletion of C3 alone nor the combined deletion of Rag1 and C3 resulted in objective histological improvement of GN or a reduction of proteinuria or splenomegaly compared with Tnip1 deletion alone, although Tnip $1^{--} \mathrm{C}^{-/-}$mice showed slightly improved survival compared with that of Tnip1-1 Rag1 ${ }^{-/}$mice. (Figure 3, A-C, and Supplemental Figure 2, C and D). Collectively, these data demonstrate that GN and other disease parameters reflected by reduced survival and splenomegaly in ABIN1-deficient mice proceed largely independently of autoreactive Abs and C3-mediated complement activation, suggesting that other mechanisms drive glomerular inflammation and tissue injury, at least during these earlier stages of mesangioproliferative GN.
PMos represent a major immune cell type in inflamed glomeruli. Given our findings that $\mathrm{T}$ and $\mathrm{B}$ cells are dispensable for the development of GN, the most likely candidates driving disease were innate immune cells. Various immune cell types, including monocytes, neutrophils, macrophages, and DCs, have been identified in diseased kidneys of patients with SLE and in mouse models (for a review, see ref. 40). To identify innate immune cell types with potential function in GN in ABIN1-deficient mice, we used a panel of Abs to identify markers that could be used (a) for identification of immune cells with glomerular localization and (b) for phenotyping and cell sorting using flow cytometry. Two markers, CD43 and F4/80, were found to be particularly informative. CD43 is expressed at high levels on T cells and PMos, whereas F4/80 is primarily expressed on tissue macrophages (41). We identified cells expressing these antigens in the kidneys of ABIN1-deficient mice, however with a marked difference in localization. Although $\mathrm{CD} 3^{+}$cells localized almost exclusively in glomeruli, $\mathrm{F} 4 / 80^{+}$cells were found primarily in the tubulointerstitium (Figure 4A and Supplemental Figure 3A). Flow cytometric analysis of single-cell populations isolated from the kidneys showed an overall increase of $C D 11 \mathrm{~b}^{+}$cells in the kidneys of ABIN1-deficient mice, which was largely due to a population of $\mathrm{CD} 43^{\text {hi }} \mathrm{F} 4 / 80^{\text {lo }} \mathrm{PMos}$ 
A

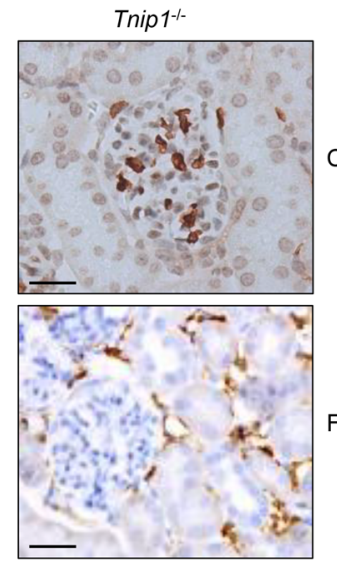

D

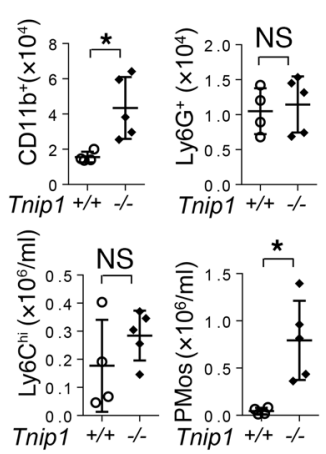

B

CD43

\section{E}
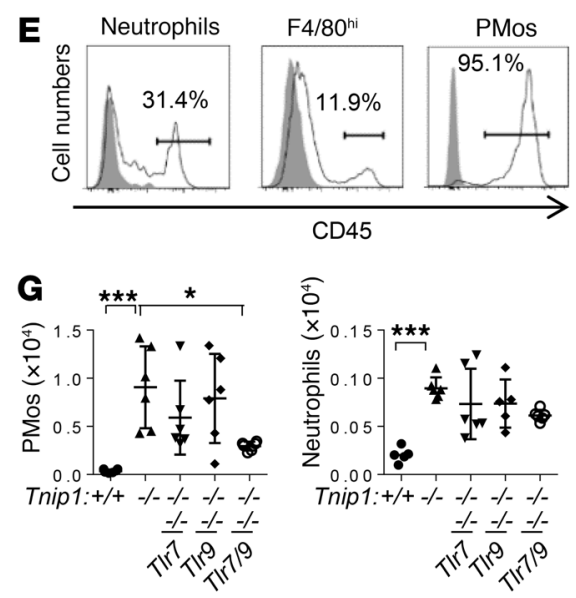

C
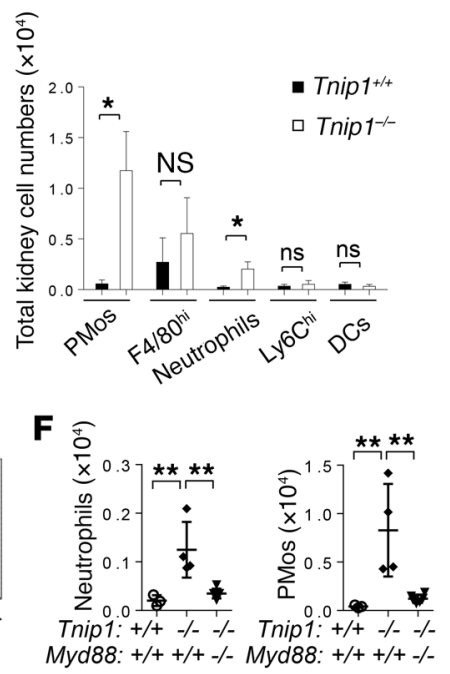

Myd88: $+/++/+-/-$ Myd88: $+/++/+-/-$

Figure 4. TLR-dependent accumulation of PMos in glomerular capillaries of Tnip1 ${ }^{-/-}$mice. (A) Kidney histology (IHC) images (scale bars: $\left.50 \mu \mathrm{m}\right)$ and (B)

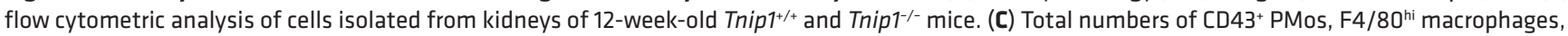

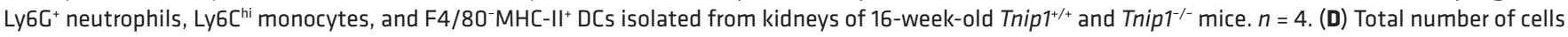
of the indicated immune cell types isolated from PB of 12-week-old Tnip $1^{+/+}$and Tnip1 $1^{-1-}$ mice as defined in Supplemental Figure 3B. (E) Flow cytometric analysis of the indicated immune cell populations isolated from kidneys of a Tnip1 ${ }^{-1-}$ mouse 10 minutes after i.v. injection of anti-CD45 Abs. This result was confirmed in a second experiment. ( $F$ and $\mathbf{G}$ ) Total number of cells of the indicated immune cell types isolated from kidneys of 16-week-old mice of the indicated genotypes. Data represent the mean \pm SD. ${ }^{*} P<0.05$, ${ }^{* *} P<0.01$, and ${ }^{* * *} P<0.001$, by Mann-Whitney $U$ test (C and $\mathbf{D}$ ) and 1 -way ANOVA with Tukey's multiple comparisons test ( $\mathbf{F}$ and $\mathbf{G}$ ). Symbols represent data from individual mice.

(Figure 4B). A more detailed flow cytometric analysis confirmed the typical phenotype of PMos ( $\left.\mathrm{CD} 43^{\mathrm{hi}} \mathrm{CD} 11 \mathrm{c}^{+} \mathrm{F} 4 / 80^{\mathrm{lo}} \mathrm{MHC}-\mathrm{II}\right)$ along with other myeloid cell types, including Ly6 $\mathrm{G}^{\mathrm{hi}}$ neutrophils, F4 $/ 80^{\text {hi }}$ macrophages, CD11c $\mathrm{F}^{+} / 80^{\mathrm{lo}} \mathrm{MHC}-\mathrm{II}^{\mathrm{hi}} \mathrm{DCs}$, and Ly6C $\mathrm{C}^{\mathrm{hi}}$ classic monocytes (Figure 4, B and C, and Supplemental Figure 3B). We note that the expression of CD11c on PMos sets these cells apart from a previously described glomerular macrophage population described in the NZM2328 model (42). While we observed a particularly marked increase of PMos in ABIN1-deficient mice, we also detected an increase in neutrophils, whereas the numbers of $\mathrm{F} 4 / 80^{\text {hi }}$ tissue macrophages, classic inflammatory Ly $6 \mathrm{C}^{\mathrm{hi}}$ monocytes, and DCs were only marginally or not increased (Figure 4C). Cell-sorting and cytology analyses confirmed the classic morphology of neutrophils $\left(\mathrm{Ly} 6 \mathrm{G}^{\mathrm{hi}}\right)$, macrophages $\left(\mathrm{F} 4 / 80^{\mathrm{hi}}\right)$, as well as the typical, relatively immature morphology of PMos $\left(\mathrm{F} 4 / 80^{\mathrm{lo}} \mathrm{CD} 43^{\text {hi }}\right)$ (25) (Supplemental Figure 3C). Just like the overall disease phenotype in ABIN1-deficient mice, the increase of PMos and neutrophils in the kidneys was due to bone marrow-derived cells (BMDCs), as revealed by BM-chimeric mice (Supplemental Figure 4A). PMo identity was further confirmed by mRNA analysis of ontogeny markers, which showed the expected high expression of Nr4a1, Cebpb, and Csfr1 (CD115) and low expression of macrophage markers such as
Fcgr1 (CD64), Mafb, Runx1, Flt3, Ccr7, and Kit (Supplemental Figure $4, \mathrm{~B}$ and C). As a further confirmation of their identity as PMos, we found that these cells were largely absent from NR4A1-deficient mice, which have a select defect in PMo generation (see below). Interestingly, increased numbers of PMos were also detected in the PB of ABIN1-deficient mice (or mice reconstituted with BM from ABIN1-deficient mice), whereas the numbers of their progenitor cells, i.e., Ly6 $\mathrm{C}^{\text {hi }}$ monocytes and neutrophils, were not affected (Figure 4D and Supplemental Figure 4, D and E) (43).

Given the established function of PMos inside of blood vessels, we investigated their topology by i.v. injection of Abs against CD45, a common leukocyte marker, followed by flow cytometric analysis. Virtually all PMos isolated from the kidneys were stained with anti-CD45 Abs, indicating their intravascular localization (Figure 4E). In contrast and consistent with their tubulointerstitial localization observed by histology, the majority of $\mathrm{F} 4 / 80^{\text {hi }}$ tissue macrophages remained $\mathrm{CD} 45^{-}$, whereas staining of neutrophils showed that approximately 50\% of the cells remained intravascularly located (Figure $4 \mathrm{E}$ ). We confirmed the glomerular localization of PMos by isolation of kidney glomeruli, which strongly enriched the frequency of PMos at the expense of $\mathrm{F} 4 / 80^{\text {hi }}$ macrophages (Supplemental Figure $4 \mathrm{~F}$ ). 
A

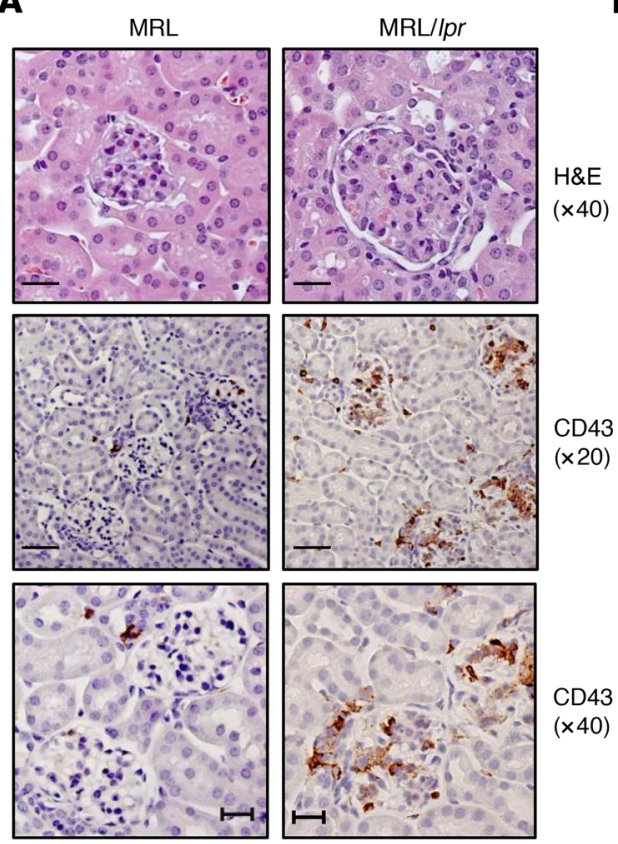

B

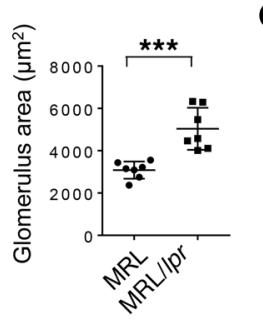

$\mathbf{E}$
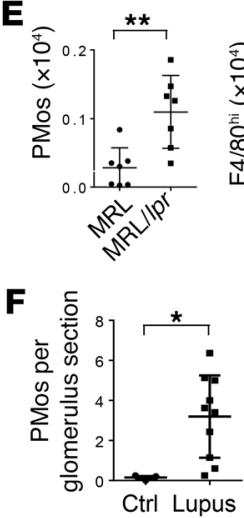
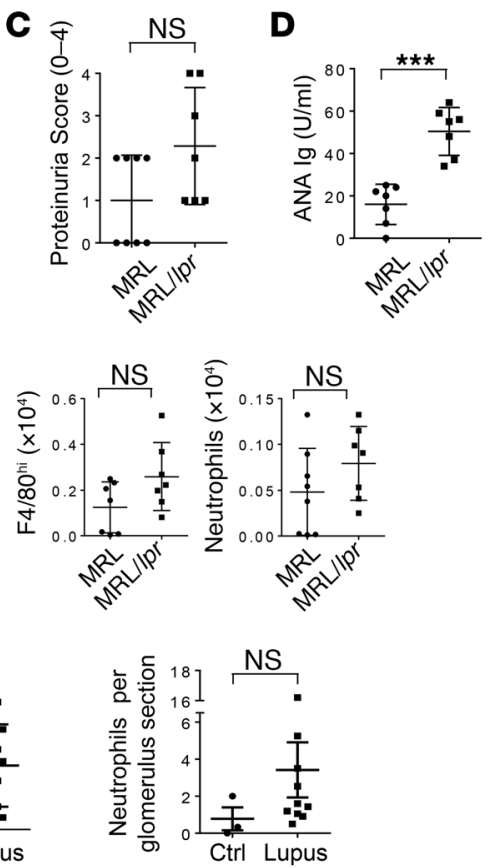

Figure 5. PMos accumulate in the glomeruli of diseased MRL/Ipr mice and patients with SLE. (A-C) Kidney pathology and function of 14-week-old MRL and $\mathrm{MRL} /$ /pr mice as analyzed by (A) histology based on H\&E and anti-CD43 Ab staining, (B) measurement of average glomerulus size, and (C) measurement of proteinuria. Representative images of glomeruli are shown. Scale bars: $20 \mu \mathrm{m}$ (original magnification, $\times 40 ; \mathrm{H} \& \mathrm{E}$ and CD43) and $50 \mu \mathrm{m}$ (original magnification, $\times 20 ;$ CD43). (D) Levels of ANA Ig in serum from the mice described in A. (E) Total number of PMos, tissue macrophages, and neutrophils isolated from kidneys of the mice described in A. (F) Numbers of CD16 ${ }^{+}$CD14-CD15- PMos and CD16-CD14-CD15+ neutrophils in glomeruli sections of reperfusion renal transplant biopsies from control patients (Ctrl) or patients with lupus nephritis (class IV-G). Data represent the mean $\pm \mathrm{SD}$. ${ }^{*} P<0.05,{ }^{* *} P<0.01$, and ${ }^{* * *} P<0.001$, by Mann-Whitney $U$ test. Symbols represent data from individual mice.

Given that GN in ABIN1-deficient mice is mediated by MyD88, we also assessed PMo and neutrophil numbers in MyD88-, TLR7and TLR9-deficient mice. As expected, Myd88 deletion and combined deletion of Tlr7 and Tlr9 reduced the accumulation of PMos and neutrophils in PB and kidneys (Figure 4, F and G). Together, our data show that the PMo is the quantitatively dominating immune cell type in the glomerular capillaries of ABIN1deficient mice and that the increased presence of PMos in PB and kidneys depends on signaling via nucleic acid-recognizing TLRs. Given their known responsiveness to nucleic acids and emerging function in vascular inflammation, PMos appear to be the prime suspects in GN.

PMos accumulate in kidney glomeruli of $M R L / l p r$ mice and patients with SLE. To determine whether PMos are also involved in GN in another mouse model, we analyzed the kidneys of MRL.Fas ${ }^{\mathrm{lpr}}\left(\mathrm{MRL} / l_{\mathrm{p} r}\right.$ ) mice, characterized by the autoimmune background of the MRL strain and a loss-of-function mutation of Fas (lpr), which drives inflammation (44). In diseased animals with GN, as determined by histology, proteinuria, and increased ANA Ig, flow cytometry revealed significantly increased numbers of PMos in the kidneys of MRL/lpr mice but not in those of the respective control mice, and IHC analysis located these cells almost exclusively in the kidney glomeruli, akin to what is seen in Tnip1 $1^{--}$mice (Figure 5, A-E). Similar data were obtained for the B6.Sleyaa model (see below).

PMos were previously reported in the glomeruli of patients with SLE $(25,30)$. To confirm these findings, we established an IHC protocol using Abs against CD16, CD14, and CD15 for the unequivocal identification of $\mathrm{CD} 16^{+} \mathrm{CD} 14^{\mathrm{dim}}$ PMos and $\mathrm{CD} 15^{+}$neutrophils and exclusion of the small population of $\mathrm{CD} 16^{+} \mathrm{CD} 14^{+}$cells, which are possibly an intermediate between classic $\mathrm{CD} 16^{-} \mathrm{CD} 14^{+}$monocytes and $\mathrm{CD} 16^{+} \mathrm{CD} 14^{\mathrm{dim}} \mathrm{PMos}(25,30)$ (see Methods and Supplemental Figure 5). We analyzed kidney samples from 10 patients with a first episode of GN [class IV-G(a)] with moderate to severe activity and minimal to no chronicity (31). We found that glomerular PMo numbers were significantly increased in the kidneys of patients with lupus, whereas neutrophil numbers appeared to be increased but not to a statistically significant degree (Figure 5F). Collectively, the data from different mouse models and patients with lupus demonstrate a correlation between glomerular PMo accumulation and GN.

Genetic deletion of ABIN1 in monocytes drives GN. The data described above indicate a possible role of monocytes, specifically PMos, in GN pathogenesis in mice with germline deletion of Tnip1/ Abin1. To determine whether more selective deletion of ABIN1 in myeloid cells, particularly those of the monocytic lineage, would recapitulate nephritis, we crossed conditional ABIN1-deficient mice (Tnip $1^{\text {fff }}$ ) (23) with LysMCre mice. LysMCre mice delete floxed genes efficiently in monocytes, macrophages, and neutrophils but only to a very small extent in DCs, and not in B or T cells (45). We confirmed deletion of ABIN1 in various cell populations by immunoblotting, which showed near-complete deletion of ABIN1 in macrophages, without affecting ABIN1 expression in DCs or B cells; ABIN1 was not expressed at measurable levels in neutrophils (Supplemental Figure 6A). Thus, crossing Tnip $1^{1 / f l}$ mice with LysMCre mice generates a model in which loss of ABIN1 function is (largely) restricted to the monocytic lineage. Consistent with a critical role of monocytes in 
A

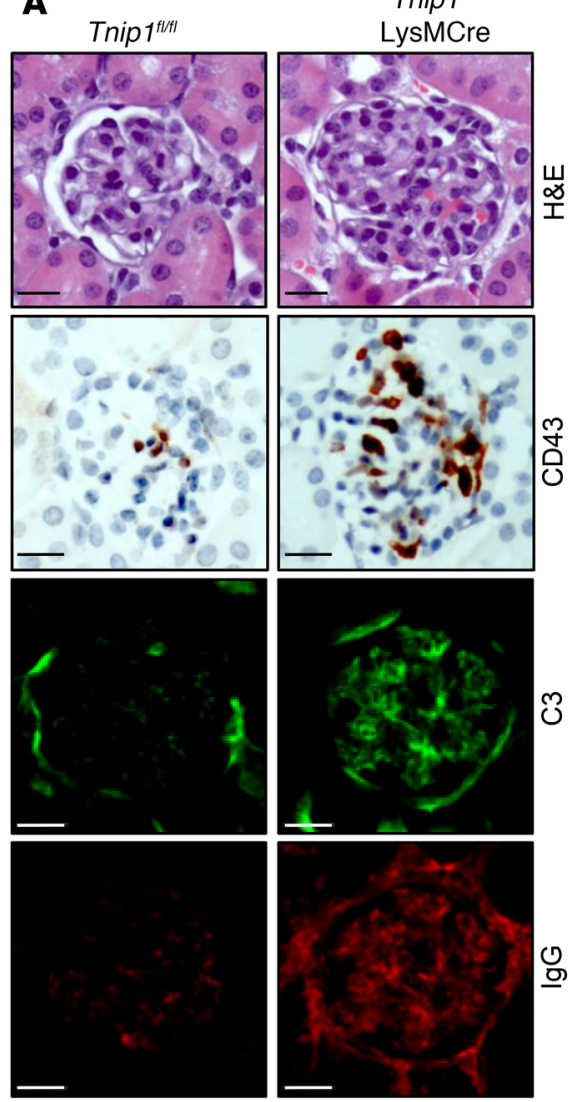

B

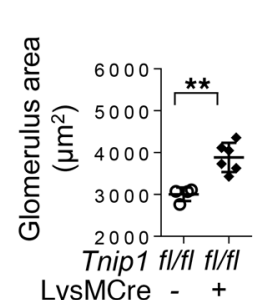

C

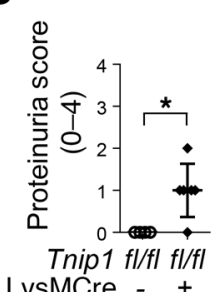

D

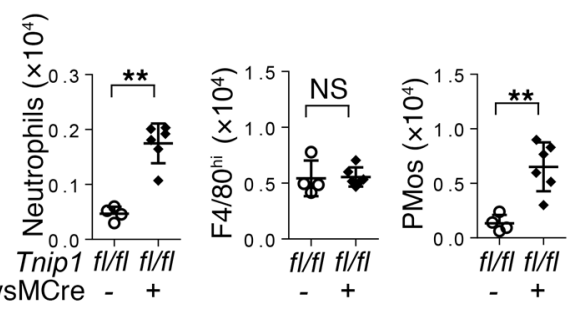

E दิ

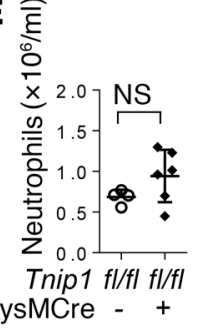

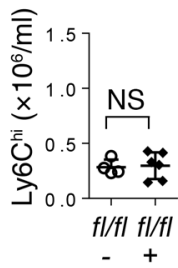

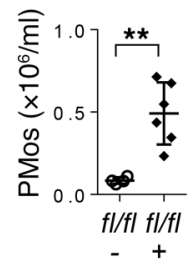

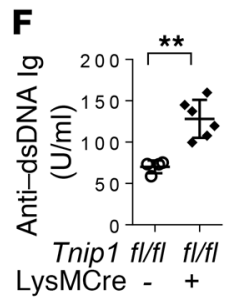

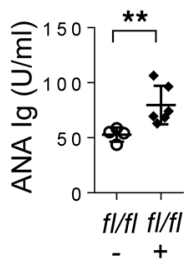

Figure 6. LysMCre-mediated deletion of ABIN1 promotes autoimmunity and GN. (A-C) Kidney pathology and function of 12-week-old Tnip $7^{f / f l}$ and Tnip $7^{f / f f I}$ LysMCre mice as analyzed by (A) histology based on H\&E staining and staining for Abs against CD43, C3, and IgG, (B) measurement of average glomerulus size, and (C) measurement of protein concentration in urine. Representative images of glomeruli are shown (scale bars: $20 \mu \mathrm{m}$ ). (D and E) Total number of cells of the indicated immune cell types isolated from (D) kidneys or (E) PB of 12-week-old Tnip fl/fl $^{f / 2}$ and Tnip ${ }^{f / f f l}$ LysMCre mice. (F) Levels of anti-dsDNA Ig and ANA Ig in serum from 12-week-old Tnip $7^{f / f l}$ and Tnip $7^{f / f l}$ LysMCre mice. Data represent the mean \pm SD. ${ }^{*} P<0.05$ and ${ }^{* *} P<0.01$, by Mann-Whitney $U$ test. Symbols represent data from individual mice.

lupus nephritis and similar to mice with germline deletion of ABIN1, Tnip $1^{\text {flfl }}$ LysMCre mice developed the major symptoms of lupus nephritis, including histologically apparent GN and proteinuria, which were also accompanied by increased numbers of $\mathrm{CD} 43^{+} \mathrm{PMos}$ in the kidney glomeruli and PB; classical Ly6 $\mathrm{C}^{\text {hi }}$ monocyte numbers were not affected (Figure 6, A-E). Neutrophil numbers in the kidneys, but not PB, were also increased in Tnip $1^{7 / P}$ LysMCre mice, most likely reflecting a consequence of deregulated monocyte biology, given the lack of ABIN1 expression in neutrophils (Figure 6, D and E, and Supplemental Figure 6A). Interestingly, these mice also displayed other symptoms of inflammatory disease observed in germline Tnip1 $1^{--}$mice, including myeloid cell infiltration into the liver, anemia, and splenomegaly, as well as reduced survival (Supplemental Figure 6, B-E). These mice also showed significantly increased levels of autoreactive Abs and glomerular IC deposits (Figure 6, A and F). Although such IC deposits are most likely irrelevant for kidney disease, as suggested by the Rag1 experiments described above, these results imply that deregulation of innate immune cell biology represents the major cause of development of the various inflammatory disease symptoms, including autoimmunity, rather than being a consequence thereof. We note that data emphasizing the role of innate immune cells as possible drivers of lupus-like inflammatory disease and autoimmunity were also obtained by CD11c-CREmediated deletion of the Src kinase LYN, most likely targeting LYN expression in DCs and PMos (46).

Given that PMos are most likely derived from Ly6C $\mathrm{C}^{\text {hi }}$ monocytes, whose numbers are not affected in ABIN1-deficient mice, it appears that ABIN1 specifically controls monocyte maturation. To determine whether this process is intrinsically (de-)regulated in developing monocytes, we used 2 independent experimental systems. In the first one, we took advantage of a recently established cell system that allows in vitro expansion of early hematopoietic progenitor cells (Hoxb8-FL cells), whose differentiation into different lymphoid and myeloid lineages, including monocytes, can be analyzed by adoptive transfer into irradiated mice (Supplemental Figure 6F) (47). Hoxb8-FL cells from WT and ABIN1-deficient BM cells were established and retrovirally transduced with vectors expressing different fluorescent proteins, allowing for adoptive transfer as mixed cell populations and precise ex vivo quantification (detailed in Methods). As shown in Figure 7, A and B, the frequency of PMos derived from ABIN1-deficient progenitors was strongly increased in the $\mathrm{PB}$ and kidneys. Retroviral reconstitution of ABIN1-deficient progenitors with ABIN1 reverted this phenotype without affecting the 
A

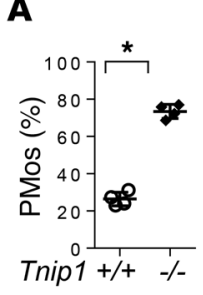

B

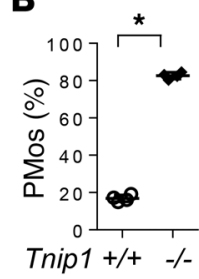

C

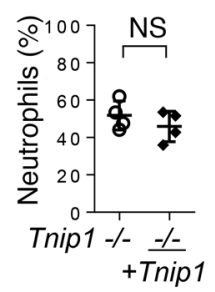

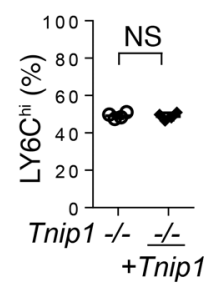

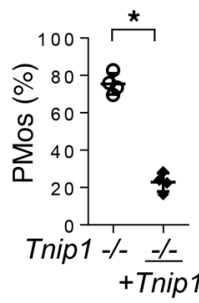

E
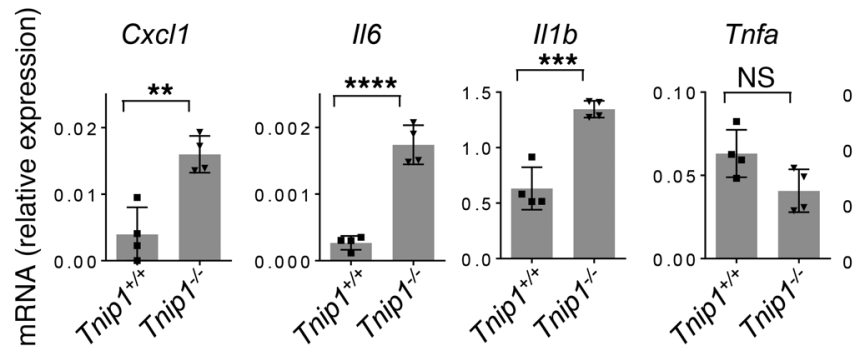

Csf3
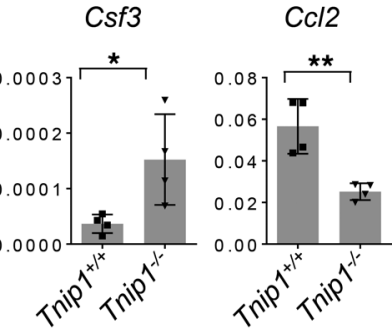

D

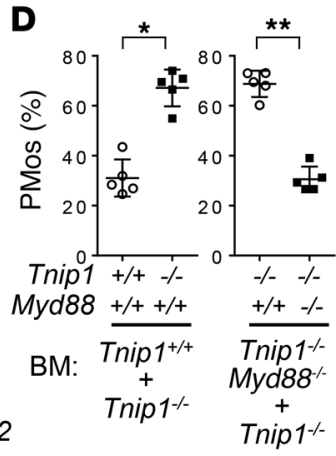

Figure 7. Cell-intrinsic function of ABIN1 controls the development and inflammatory phenotype of PMos. (A and B) Relative numbers of CD43 ${ }^{+}$PMos derived from Tnip1 $1^{+/}$and Tnip1 $1^{-/-}$Hoxb8-FL cells in (A) PB and (B) kidneys 11 days after adoptive transfer of Hoxb8-FL cells (detailed in Methods). (C) Relative numbers of Tnip1 1- and Tnip1-reconstituted (+ Tnip1) Ly6C+ neutrophils (left), Ly6C ${ }^{\text {hi }}$ monocytes (middle), and CD43+ PMos (right) derived from the Hoxb8-FL cells described in A. Data on cells isolated from kidneys 8 days after adoptive transfer of Hoxb8-FL cells are shown. (D) Relative numbers of

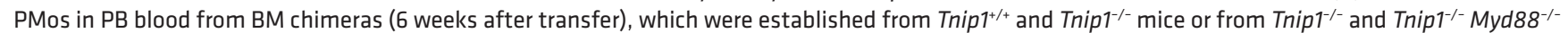
mice as indicated. (E) Quantitative PCR analysis of PMos that were FACS sorted from kidneys of BM-chimeric mice, based on BM from Tnip $7^{+/+}$and Tnip ${ }^{1 /-}$ mice as indicated (25 weeks after BM transfer). Data represent the mean $\pm \mathrm{SD}$. ${ }^{*} P<0.05,{ }^{* *} P<0.01,{ }^{* * *} P<0.001$, and ${ }^{* * * *} P<0.0001$, by Mann-Whitney $U$ test $(\mathbf{A}-\mathbf{D})$ and unpaired $t$ test $(\mathbf{E})$. Symbols represent data from individual mice.

generation of Ly6 $\mathrm{C}^{\text {hi }}$ monocytes or neutrophils (Figure 7C). Given that both cell populations were transferred into the same animal, these data suggest that ABIN1 controls the numbers of PMos in a cell-intrinsic manner, either by regulating cell maturation or the half-life of PMos. We confirmed and extended these findings using mixed BM chimeras of either Tnip $1^{+/+}$and Tnip $1^{-/-} \mathrm{BM}$ or Tnip $1^{-/}$and $M y d 88^{--}$Tnip1 $1^{--}$BM. Although the total cell numbers and major cell lineages derived from Tnip1 ${ }^{-/}$BM including classic monocytes, $\mathrm{B}$ cells, and $\mathrm{T}$ cells were reduced, possibly reflecting a reduction of hematopoietic stem cells in the transferred BM from inflamed mice, the frequencyof Tnip1 ${ }^{--}$PMos was strongly upregulated, confirming the select, cell-intrinsic upregulation of PMos due to a loss of ABIN1 function (Figure 7D and Supplemental Figure 7). Of note, Myd88 deletion in Tnip1 $1^{--}$cells reverted this PMo phenotype completely and also rescued the efficacy of the overall reduced BM transfer of Tnip1 $1^{-/}$cells (Figure 7D and Supplemental Figure 7). Given the causal link between MyD88 expression and PMo deregulation, we tested whether genes with inflammatory function might be deregulated in ABIN1-deficient PMos. Indeed, $I l 6, I l 1 b, C s f 3$, and Cxcl1 were upregulated in ABIN1-deficient PMos, the latter of which has been shown to control PMomediated neutrophil recruitment (Figure 7E) (48). We note that these 4 genes are known target genes of the transcription factor $\mathrm{C} / \mathrm{EBP} \beta$, which was found to be deregulated in ABIN1-deficient macrophages during TLR activation (22). These results and the data obtained from the monocyte-specific deletion experiments indicate that ABIN1 controls TLR (MyD88) signaling in PMos in a cell-intrinsic manner and that this signaling mediates inflammatory gene expression and deregulates PMo biology.
Genetic depletion of PMos protects against GN. To directly address the question of whether PMos are pathogenically relevant for GN in ABIN1-deficient mice, we used $\mathrm{Nr}_{4} \mathrm{a1}^{-/-}$mice, which are defective

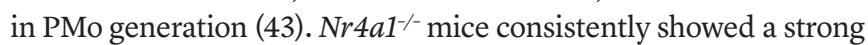
reduction of PMosin the PB, which wasmaintained in Tnip1 $1^{-/} \mathrm{Nr}_{4} \mathrm{al}^{-1-}$ mice (Supplemental Figure 8A). Given that NR4A1 plays additional roles outside the hematopoietic system (49), we focused primarily on chimeric mice that were generated using mice with a WT recipient background.Importantly, the reduction of PMonumbersupon $\mathrm{Nr} 4 \mathrm{a1}$ deletion resulted in significant protection from kidney pathology as assessed by histology and proteinuria measurement (Figure 8, A-D). The loss of PMos in the kidneys of Tnip1 ${ }^{-/} \mathrm{Nr}_{4 a 1^{-/}}$mice was accompanied by a reduction of neutrophil numbers, consistent with the interpretation that neutrophil accumulation is dependent on PMos (Figure 8D). In contrast, neither autoreactive $\mathrm{Ab}$ generation nor IgG or C3 deposition in the kidneys was affected by Nr4a1 deletion, suggesting that ICs are neither necessary nor sufficient for the development of GN, at least during these relatively early phases of disease (Figure 8, A and E). In qualitative terms, the same results were obtained with germline $\mathrm{Tnip1}^{-1-} \mathrm{Nr}^{-11^{-/}}$mice, suggesting that NR4A1 function outside the hematopoietic system is less relevant in this model (Supplemental Figure 8, B-G). Similar to the generation of autoreactive antibodies, other disease parameters such as anemia, splenomegaly, and overall survival were not affected by Nr4a1 deletion, highlighting the selectively pathogenic role of PMos in GN (Supplemental Figure 8, H-J).

To determine whether PMo are also functionally involved in GN in another mouse model, we analyzed B6.Slelyaa mice, which have the Sle 1 locus of NZM2410 mice (on a congenic C57Bl/6 background) 

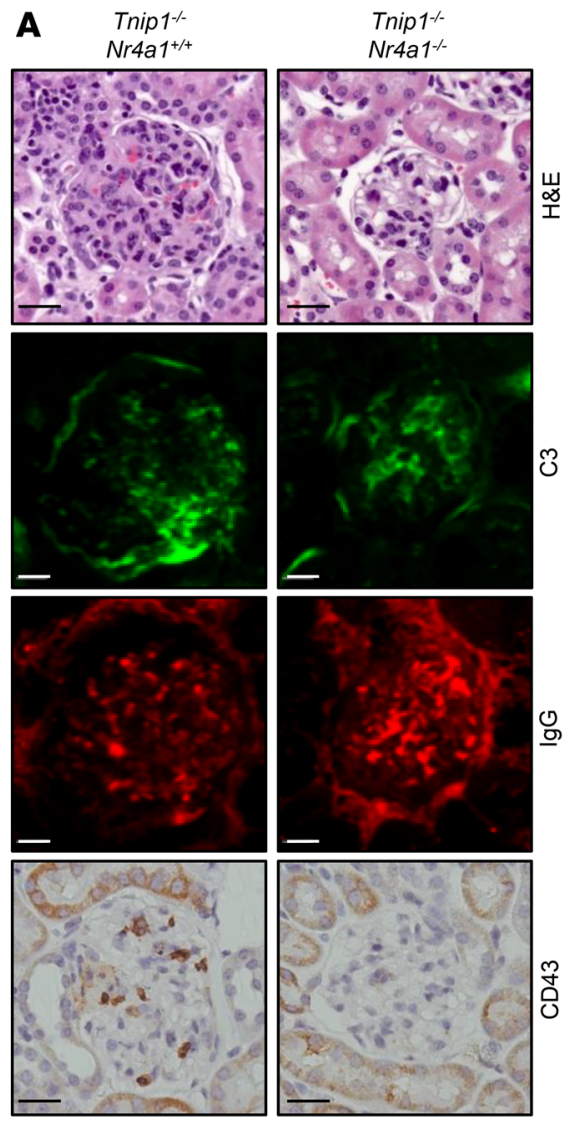

B

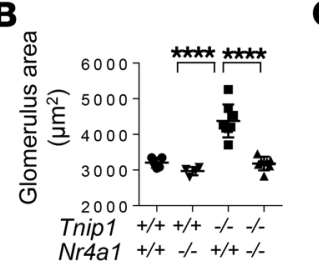

D

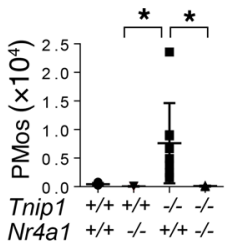

E

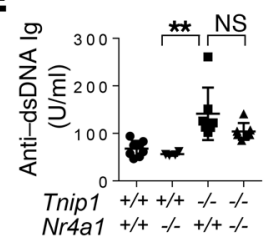

C
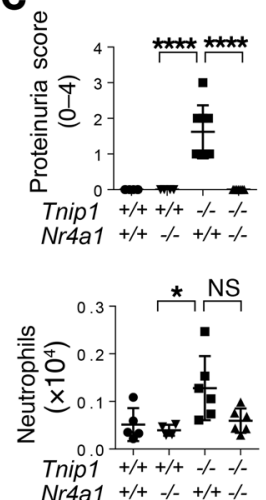

Nr4a1 +/+ - + + +/+-/-

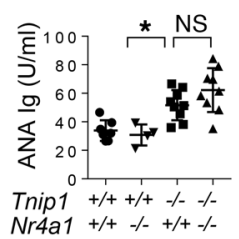

Figure 8. Genetic deletion of PMos protects against GN. (A-C) Kidney pathology and function of chimeric mice 16 weeks after transfer ( Tnip $^{1^{+/}}$recipient mice were reconstituted with fetal liver cells from mice of the indicated genotypes) as analyzed by (A) histology based on $\mathrm{H} \& \mathrm{E}$ staining and staining for Abs against C3, IgG, and CD43, (B) measurement of average glomerulus size, and (C) measurement of protein concentration in urine. Representative images of glomeruli are shown (scale bars: $20 \mu \mathrm{m}$.). (D) Total number of CD43+ PMos and $\mathrm{Ly}_{\mathrm{G}} \mathrm{G}^{+}$neutrophils isolated from kidneys of the BM-chimeric mice described in $\mathbf{A}$. (E) Levels of anti-dsDNA IgG and ANA Ig in sera from the $B M$-chimeric mice described in $\mathbf{A}$. Data represent the mean $\pm \mathrm{SD} .{ }^{*} P<0.05,{ }^{* *} P<0.01$, and ${ }^{* * * *} P<0.0001$, by 1 -way ANOVA with Tukey's multiple comparisons test. Symbols represent data from individual mice. and a gain of function (duplication) of the Tlr7 (yaa) gene (9). Flow cytometric analysis revealed significantly increased numbers of PMos in the kidneys of diseased animals but not in those of the respective control mice, and IHC analysis showed that these cells were almost exclusively located in kidney glomeruli, as is seen in Tnip1 $1^{--}$and $\mathrm{MRL} / \mathrm{lpr}$ mice (Figure 9, A and B). As with other mouse models, we found that disease was accompanied by an increase in glomerulus size and elevated levels of autoreactive Abs (Figure 9, C and D). Of note, genetic deletion of $\mathrm{Nr} 4 a 1$ in B6.Sle1yaa mice prevented GN but did not affect the serum concentration of autoreactive Abs or their glomerular deposition, suggesting an autoimmunity-independent pathogenic function of PMos, similar to what was observed in the Tnip1 model (Figure 9, A and D). Collectively, data obtained from 3 independent lupus models and human patients with lupus demonstrate an accumulation of PMos in diseased kidney glomeruli, and genetic suppression of PMos strongly suggests a pathogenic function. At the same time, the data confirm that autoimmunity, as reflected by the production of autoreactive Abs and glomerular IC deposits, and end-organ damage, as reflected by kidney pathology, can proceed independently of each other.

\section{Discussion}

This work provides evidence that PMos and nucleic acid-recognizing TLRs play critical roles in lupus nephritis due to loss of ABIN1 function. Consistent with a more general role in lupus nephritis, PMos also accumulate in the kidney glomeruli of MRL/lpr and B6.Sle1yaa mice and in patients with SLE. As mentioned above, increased numbers of PMos in the glomeruli of patients with SLE have been reported in other studies $(25,30)$. Although those studies were limited in scope (25) or did not exclude CD16 ${ }^{+} \mathrm{CD} 14^{+}$cells (30), our data confirm their findings. We also excluded a significant contribution of $\mathrm{CD} 16^{+} \mathrm{CD} 14^{+}$ cells, thereby validating the earlier report by Yoshimoto et al., which showed a correlation of glomerular $\mathrm{CD}^{1} 6^{+}$monocyte numbers (PMos) and GN severity and an inverse correlation of glomerular PMos with the therapeutic response. As such, the number of PMos in kidney glomeruli correlated with GN. As demonstrated in the Tnip1 and B6.Sle1yaa models, genetic suppression of PMos ameliorated GN, suggesting a pathogenic function of these cells. In both models, PMos promoted GN independently of autoreactive Abs, which were neither necessary nor sufficient to cause GN, as was apparent in the RAG1- and NR4A1-deficient mice. These data are consistent with other work in MRL/lpr mice, in which Ig secretion and IC formation were genetically prevented (38), as well as work based on inhibition of the PMo-attracting chemokine CX3CL1, which blocked monocyte accumulation and ameliorated GN but did not affect the generation of autoreactive $\mathrm{Abs}(30,50)$. The data are also consistent with observations in siblings of patients with lupus, who may have autoreactive Abs but no GN symptoms (51). Together, it appears that PMos can mediate GN independently of autoreactive Abs, at least during the early phase of disease, which we investigated in this work. Autoreactive Abs and IC may, dependent on their titer and antigen specificity, contribute to pathology in the context of specific susceptibility loci or, alternatively, become more relevant during later stages of the disease, in particular extraglomerular pathology possibly coordinated by kidney-resident macrophages (52). Splenomegaly and survival, like autoreactive Abs, were not rescued by NR4A1-mediated PMo 
A
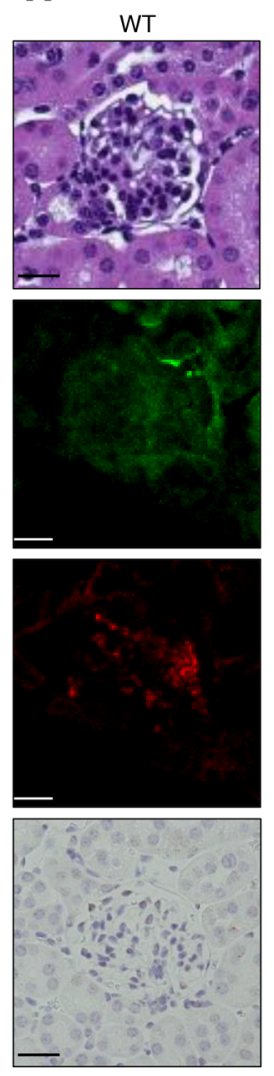
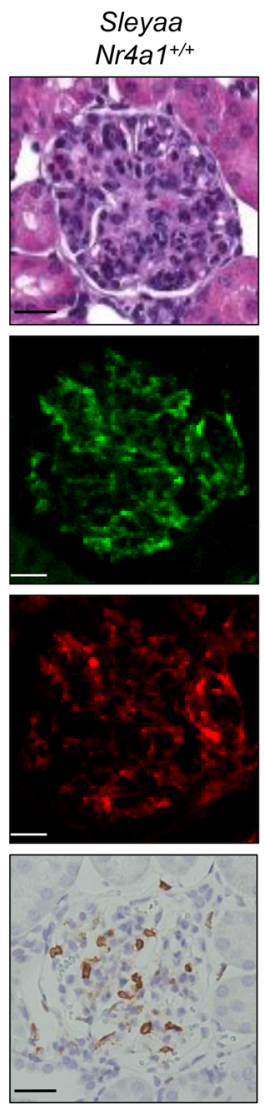
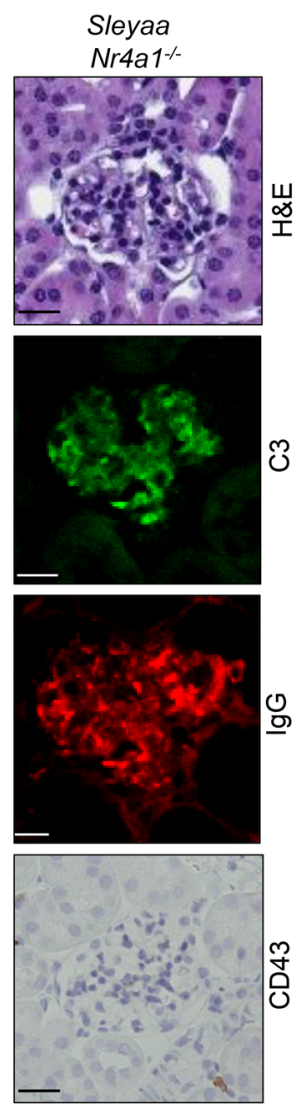

B
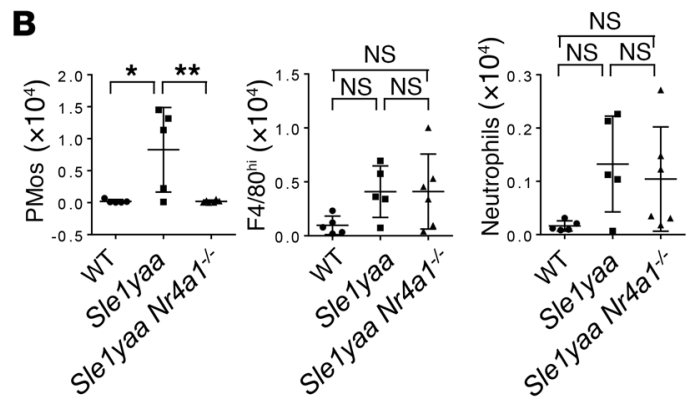

C
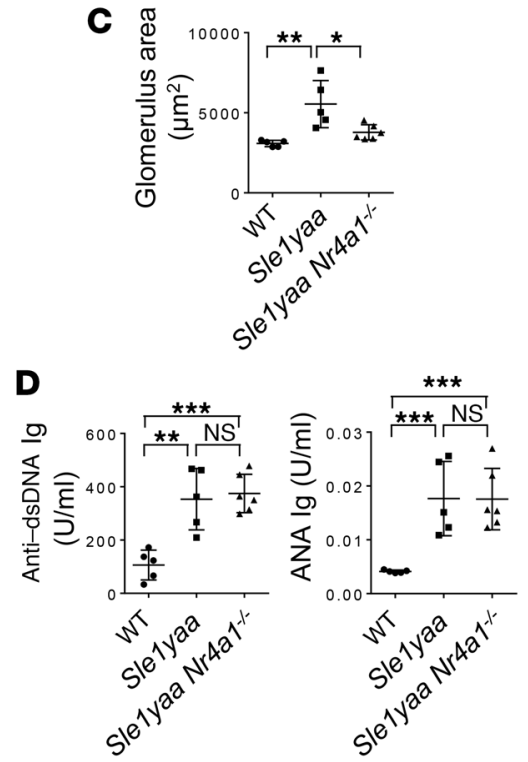

Figure 9. PMos promote GN in B6.Sleyaa mice independently of autoimmunity. (A) Kidney pathology of 14-week-old WT, B6.Sleyaa, and B6.Sleyaa Nr4a1 $/$ mice as analyzed by histology based on H\&E staining and staining for Abs against C3, IgG, and CD43. Representative images of glomeruli are shown (scale bars: $20 \mu \mathrm{m}$ ). (B) Total number of PMos, tissue macrophages, and neutrophils isolated from kidneys of the mice described in $\mathbf{A}$. (C) Average size of kidney glomeruli and (D) serum levels of anti-dsDNA IgG and ANA Ig of the mice described in $\mathbf{A}$. Data represent the mean \pm SD. ${ }^{*} P<0.05,{ }^{*} P<0.01$, and ${ }^{* *} P<0.001$, by 1-way ANOVA with Tukey's multiple comparisons test. Symbols represent data from individual mice.

suppression. Given that innate immune cells drive disease, as observed in $\mathrm{Ragl}^{-/-}$mice and with LysMCre-mediated ABIN1 deletion, it appears that 3 largely independent effector mechanisms can be differentiated, i.e., PMo-mediated GN, autoimmunity, and systemic inflammation, the latter of which may be mediated primarily by the LysMCre-targeted monocyte/macrophage lineage (Figure 10). Consistent with an important role of this lineage, treatment of MRL/lpr mice with a small-molecule inhibitor of macrophage-CSF receptordriven (M-CSF receptor-driven) monocyte/macrophage generation ameliorated kidney pathology and neuropsychiatric disease, without affecting autoreactive Ab titers; other parameters including splenomegaly and survival were not assessed in that study (53).

Although our work showed that PMo suppression based on $\mathrm{Nr} 4 \mathrm{a} 1$ deletion in hematopoietic cells provided protection from GN, indicating NR4A1 as a possible therapeutic target (Figure 6), we note that $\mathrm{Nr} 4 \mathrm{a} 1$ deletion in fibroblasts was shown to promote TGF- $\beta$-mediated fibrosis in various models and also worsened kidney disease, in particular with tubulointerstitial symptoms, in the fawn-hooded hypertensive rat model $(49,54)$. Likewise, animal models of strictly $\mathrm{T}$ cell-dependent autoimmunity, such as those for experimental autoimmune encephalomyelitis (EAE), allergic contact dermatitis, and collagen-induced arthritis, revealed a protective function of NR4A1 (55). As such, it seems likely that physiological functions unrelated to PMo biology limit the viability of NR4A1 as a therapeutic target.

Different experimental settings demonstrated that ABIN1 controls PMo biology in a cell-intrinsic manner, and BM-chimeric mice indicate that TLR (MyD88-mediated) activation of PMos is responsible for deregulation. A similar mechanism may be at play in the B6.Sle1yaa mouse model, in which disease is promoted by increased TLR7 activity (9). Thus, an important question is how TLR-activated PMos mediate GN. One possibility is that increased cell numbers and cumulative dwell time in the glomeruli suffice to instigate glomerular dysfunction and inflammatory damage. This interpretation is supported by estimates based on intravital imaging studies and the approximately 20-fold increase of PMos measured in the kidneys of Tnip1/-- mice, predicting more than 1 PMo per glomerular capillary ( $20 \mathrm{PMo} / 4-8$ parallel capillaries per glomerulus) at any given time (27). Given the diameter of monocytes $(20 \mu \mathrm{M})$ and capillaries $(\sim 8 \mu \mathrm{m})$, it seems likely that basic glomerular functions such as proper blood flow will be affected by pathologically increased PMo numbers. Another possibility is that GN is promoted through specific, PMo-dependent effector functions that need to be considered in context with the more recent but rapid appreciation 


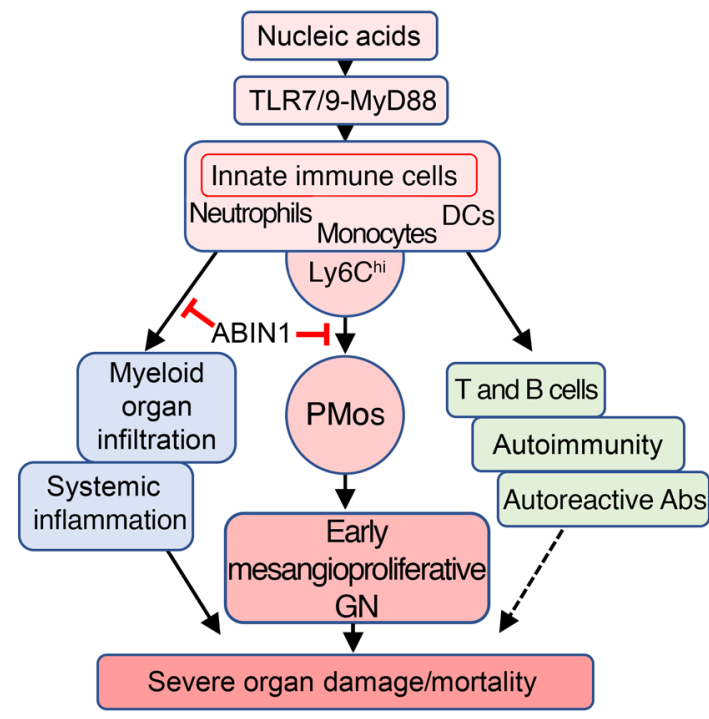

Figure 10. Model of lupus-like inflammatory disease pathogenesis in Tnip1 ${ }^{-1-}$ mice. The hierarchy of inflammatory events shown is based on the following observations: (a) Genetic deletion of MyD88 or combined deletion of TLR7 and TLR9 largely protected from all analyzed disease parameters. (b) LysMCre-mediated deletion of ABIN1 in monocytes recapitulated all analyzed disease parameters, including the generation of autoreactive AB. (c) Adoptive transfer models using Hoxb8-FL cells or BM suggest a cell-intrinsic function of ABIN1 and MyD88 in the monocyte lineage. (d) On the basis of experiments using Rag1- and Nr4a1-deficient mice, it appears that deregulation of the adaptive immune system, including the generation of autoreactive $A B$ and IC, is neither sufficient nor necessary for promotion of the early phase of mesangioproliferative $\mathrm{GN}$ (but may contribute to later stages of $\mathrm{GN}$ or other disease symptoms).

of the PMo as a specialized macrophage cell type that guards the integrity of blood vessels through orchestration of an inflammatory response. Although the experimental settings used in related studies focused on leukocyte biology during acute inflammatory insults and, in part, on capillaries outside the glomeruli, it is likely that key events of the observed multistep process follow common principles. Exposure of kidneys to a TLR7 agonist or injection of anti-glomerular basement membrane (anti-GBM) Abs triggered endothelial PMo retention (dwelling), which provided the basis for CXCL1-mediated recruitment and interaction with neutrophils, the latter of which caused endothelial cell damage $(26,27)$. Data on Tnip1 $1^{-/}$mice are compatible with the described sequence of events, where (a) PMos exhibited increased CXCL1 expression; (b) neutrophils were found in the kidney glomeruli but not the PB of Tnip1 $1^{-1}$ mice, consistent with their PMo-dependent glomerular recruitment; (c) select deletion of ABIN1 in monocytes led to accumulation of neutrophils in glomeruli; and (d) genetic PMo suppression prevented glomerular neutrophil accumulation. As such, glomerular neutrophil accumulation appears to be a consequence of deregulated PMo function. Of note, a pathogenic role of neutrophils in SLE is substantiated by data from human patients and mouse models (56-59). Together, it seems plausible that both increased PMo numbers and PMo-instigated inflammatory consequences, including neutrophil recruitment and cytotoxicity, cooperate to promote GN in Tnip1 $1^{--}$mice and probably in other mouse models and human SLE (57).
In summary, our observations from ABIN1-deficient mice, various mouse models, and human patients with SLE are compatible with a scenario in which PMo-mediated vascular inflammation is critical for end-organ damage and GN. A major question, given these findings, is how the various genetic and environmental factors driving lupus nephritis interface with PMo biology, including the complex multistep process, the complex multistep process that entails PMo maturation, endothelial cell signaling, PMo recruitment and activation, neutrophil recruitment and activation, endothelial cell death, and removal of immunostimulatory debris. Given the multiple possibilities of deregulation of this process, it seems likely that various factors instigate GN independently of autoimmunity, as was seen with loss of TNIP1 function. Other factors, e.g., genetic alterations of Fcreceptors and ITGAM, which are intimatelyinvolved in Ig- and complement-mediated immunity, respectively, may drive $\mathrm{GN}$ as a consequence of autoimmunity and Ig/C3-mediated functions (60). With these considerations in mind, one important opportunity for lupus research will be to assign the various SLE susceptibility factors to specific aspects of PMo-directed vascular inflammation in order to define common and unique mechanisms. This information may reveal novel therapeutic options and could be critical for stratifying patients for specific clinical trials.

\section{Methods}

Mice and cell culture. With the exception of MRL/lpr mice, all mice used in this study were on a C57BL/6 background. Tnip1 ${ }^{-/}$mice were backcrossed with mice on a C57BL/6 background for more than 10 generations and have been described previously (22).

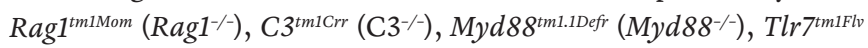

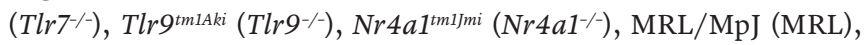
MRL/MpJ-Faslpr/J (MRL/lpr), and B6.Cg-Sle1 ${ }^{\text {NZM2410/Aeg Yaa/DcrJ }}$ (B6.SLE1yaa) mice were purchased from The Jackson Laboratory $(9,37,61-65))$. Tnip $1^{7 / / A}$ mice were described previously (23) and crossed with LysM-Cre (Lyz $\left.2^{\text {tml(crelffo }}\right)$ mice established by Förster et al. (45) and obtained from The Jackson Laboratory. Age- and sex-matched littermates at 12 to 16 weeks of age were used for all experiments, unless otherwise indicated. BM cells were obtained by flushing from femurs and tibiae of mice. For the generation of BM-chimeric mice, $1 \times 10^{6}$ cells in $200 \mu \mathrm{lBS}$ containing $1 \% \mathrm{FBS}$ were transferred via tail-vein injection into recipient mice that had been lethally irradiated at $9.5 \mathrm{~Gy} 1$ day before cell transfer. For fetal liver transplantation, $1 \times 10^{6}$ fetal liver cells isolated from Tnip1 $1^{+/+}$,

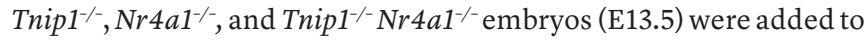
$200 \mu \mathrm{l} \mathrm{PBS}$ containing $1 \% \mathrm{FBS}$ and transferred via tail-vein injection into recipient mice that had been lethally irradiated at $9.5 \mathrm{~Gy} 1$ day before cell transfer. Recipient mice (both fetal liver transplant recipients and BM-chimeric mice) were treated with antibiotics ( $2 \%$ SulfaTrim; Hi-Tech Pharmacal) in the drinking water for 5 to 6 weeks, starting 1 week before irradiation. Chimeric mice were analyzed 12 weeks after cell transfer.

For the generation of BM-derived macrophages (BMMs) and BMDCs, BM cells were cultivated in Petri dishes for 7 days in standard growth medium (DMEM; Thermo Fisher Scientific) for macrophages or RPMI 1640 (Thermo Fisher Scientific) for DCs, supplemented with $10 \%$ (v/v) FCS (Hyclone), 50 mM 2-mercaptoethanol, and antibiotics (100 IU/ml penicillin $\mathrm{G}$ and $100 \mathrm{IU} / \mathrm{ml}$ streptomycin sulfate; Thermo Fisher Scientific) containing 30\% 
L cell-conditioned medium (for BMMs) or 2\% GM-CSF (for BMDCs) derived from growth factor-producing cell lines. The $2 \%$ GM-CSF corresponded to the bioactivity of $7 \mathrm{ng} / \mathrm{ml}$ recombinant GM-CSF (BD Biosciences).

Renal biopsies. Human samples were selected from biopsies received over a 2-month period at Arkana Laboratories. Selection criteria for lupus nephritis included a first episode of lupus nephritis with a diagnosis of diffuse lupus nephritis, class IV-G (a), with moderate to severe activity and minimal to no chronicity by International Society of Nephrology/Renal Pathology Society (ISN/RPS) classification (66). Controls were timezero reperfusion biopsies from deceased donor renal transplants with no abnormalities, specifically no acute tubular injury and no chronicity.

Histology, IHC, and immunofluorescence of mouse tissues. Mice were perfused with $10 \mathrm{ml}$ PBS through the left ventricle after incision of the lateral left lobe of the liver. For histological analyses based on H\&E, PAS, JMS staining and IHC, kidneys were fixed with $10 \%$ phosphatebuffered formalin and embedded in paraffin. Deparaffinized sections of $4-\mu \mathrm{m}$ thickness were stained with H\&E (Richard-Allan Scientific) on a Dako Coverstainer (Agilent Technologies), with PAS and JMS (Dako) on the Artisan Link Stainer (Agilent Technologies), or stained with Abs against Ki67 (clone SP6; Thermo Fisher Scientific), F4/80 (clone BM8; Thermo Fisher Scientific), or CD43 (clone S7; BD Biosciences), followed by detection with secondary biotinylated Abs and HRP-labeled streptavidin (Thermo Fisher Scientific) and counterstaining with hematoxylin (Shandon; Thermo Fisher Scientific). To determine the glomeruli area range for each model, images of H\&Estained sections were acquired on an automated Zeiss Axio Scan Z.1 using a $20 \times 0.8$ objective and analyzed using Zeiss Zen Blue software or, alternatively, captured with an Aperio ScanScope XT Slide Scanner (Leica Biosystems), resulting in scalable whole slide images that were also manually outlined and analyzed using Aperio ImageScope software. The average glomerular area was determined by measuring 30 glomeruli on each H\&E-stained kidney section.

For immunofluorescence (IF) of IgG and C3, kidneys were fixed in $4 \%$ paraformaldehyde for 24 hours prior to cryoprotection using $20 \%$ sucrose in PBS. Tissues were cryosectioned and allowed to air-dry for 30 minutes before blocking nonspecific binding with $1 \%$ BSA and $0.05 \%$ Tween-2O in PBS, pH 7.4, for 1 hour at room temperature. Sections were incubated overnight at $4^{\circ} \mathrm{C}$ in blocking buffer containing FITC-conjugated anti-C3 (1 $\mathrm{g} / \mathrm{ml}$; clone RmC11H9; Cedarlane) and CR568-conjugated goat anti-mouse IgG $(1 \mu \mathrm{g} / \mathrm{ml}$; catalog 20301; Biotium). Slides were washed in PBS before mounting with HardSet media containing DAPI (Vector Laboratories). Widefield fluorescence microscopy was performed using a motorized Nikon TiE inverted microscope equipped with a $\times 20$ Plan Apo 0.75 NA objective, standard DAPI, FITC, and TRITC filter sets, and an EMCCD camera (Andor). A series of $10 \times 10$ images acquired using a $\times 20$ objective was stitched on the basis of DAPI fluorescent signal, and the subsequent large stitched images were analyzed using NIS Elements software (Nikon Instruments). High-resolution images were acquired using a Marianis spinning-disk confocal microscope (Intelligent Imaging Innovations) equipped with a $\times 401.3$ NA objective and EMCCD camera and analyzed using Slidebook software (Intelligent Imaging Innovations).

For multicolor IF analysis, formalin-fixed, paraffin-embedded (FFPE) tissues were sectioned at 4- $\mu \mathrm{m}$ thickness, mounted on positively charged glass slides (Superfrost Plus; Thermo Fisher Scientific), and dried at $60^{\circ} \mathrm{C}$ for 20 minutes. Both IHC and singleplex IF labeling methods (for
CD45, Ki67, and DESMIN) were tested and compared to determine the Ab titrations for each protein marker to be used in the multiplex IF studies. All tissue sections used for IF labeling underwent antigen retrieval in a prediluted cell-conditioning solution (CC1) (Ventana Medical Systems) for 32 minutes before the serial application of the primary Abs, using the U Discovery 5-Plex IF procedure as previously described (67). Coverslips were mounted onto slides with Prolong Gold Antifade Reagent containing DAPI (Thermo Fisher Scientific). The following Abs were used for multicolor IF: CD45 (30-F11; BD Biosciences; 1:500 dilution; CY5), Ki67 (Thermo Fisher Scientific; 1:50 dilution; FAM), and DESMIN (rabbit polyclonal, RB-9014-S; Thermo Fisher Scientific; 1:500 dilution; Red 610). Images were acquired with the Leica TCS SP8 confocal microscope using a high-contrast, plan apochromatic, $0.75-\mathrm{NA}, \times 40$ magnification objective lens. Scan lines were averaged 3 times per frame, and 3 frames were accumulated per image. Analyses of Ki67 ${ }^{+}$cells were done following image thresholding and segmentation using Imaris software (Bitplane).

Pathology scoring of mouse kidney sections. Tissue lesions related to GN, interstitial nephritis, and vasculitis/perivasculitis were graded on a $0-5$ scale based on the intensity and extent of histopathological changes. The grades $0-5$ were converted to semiquantitative scores, which were used to calculate a mean histopathological score for each mouse. Gradebased conversion of semiquantitative scores was as follows: $0=0 ; 1=1$; $1.5=8 ; 2=15 ; 2.5=25 ; 3=40 ; 3.5=60 ; 4=80 ; 4.5=90 ;$ and $5=100$. For GN determination, grading criteria were applied to 20 to 30 glomeruli: $\mathrm{O}=$ no glomerular lesions detected; 1 = minimal thickening/increased mesangium; 2 = noticeably increased mesangial and glomerular capillary cellularity; 3 = previous lesions plus detectable inflammatory exudates and capsular adhesions; $4=$ obliteration of more than $70 \%$ of the glomerular architecture; 5 = effacement of the glomerular architecture (Supplemental Figure 1).

IHC of human tissue. In the initial IHC staining step, FFPE tissue that was cut at $4-\mu \mathrm{m}$ thickness and mounted onto charged glass slides was dewaxed by placing in a $60^{\circ} \mathrm{C}$ oven for 30 minutes and then deparaffinized in 3 changes of xylene for 3 minutes each, followed by rehydration in a series of graded alcohols into distilled water. Other than heat-induced epitope retrieval (HIER), all incubation steps were performed at room temperature in a humidity chamber (manual staining). HIER was performed at $90^{\circ} \mathrm{C}$ with high pH buffer (Dako; catalog DM828) for 15 minutes in a Dako PT-Link. Endogenous peroxidase was quenched by treatment with peroxidase blocker (Dako; catalog SM801) for 5 minutes at room temperature. The sections were washed in FLEX buffer (Dako; catalog DM831). The sections were then washed and incubated with a primary Ab targeting CD15 (Abcam; catalog Ab218403) for 1 hour at room temperature. The sections were washed with FLEX buffer and then incubated with an HRP-linked secondary Ab (Dako EnVision FLEX; catalog SM802) for 20 minutes at room temperature. Next, the sections were washed in FLEX buffer at room temperature, incubated with EnVision FLEX DAB (diaminobenzidine) plus chromagen (Dako catalogs DM827 and SM803) for 10 minutes at room temperature, and then washed with FLEX buffer. The CD15-stained sections were then incubated with the primary rabbit Ab against CD14 (Sino Biological; catalog 10073R001) and a mouse Ab against CD16 (Novacastra; Leica Biosystems; catalog NCL-L-CD16) for 2 hours at room temperature. The sections were washed with FLEX buffer and then incubated with a cocktail of the secondary Abs against mouse CY2 (The Jackson Laboratory; catalog 115-225-146) and rabbit CY3 (The Jackson Laboratory; catalog 
111-165-144) for 30 minutes at room temperature. Stained sections were washed with FLEX buffer, air-dried, and then coverslipped with aqueous fluorescence mounting media containing DAPI (Dako) for viewing by fluorescence and light microscopy. After microscopic analysis of the sections (detailed below), the coverslip was removed and reacted with PAS using standard techniques.

All stains were validated in a pilot study using human tonsil as a control. The experimental and control group cells were then stained and examined using $1 \mathrm{Ab}$ alone, then $2 \mathrm{Abs}$, and, finally, all $3 \mathrm{Abs}$. This was done in order to validate the intensity and maintenance of intensity of the fluorochrome and the IHC reaction throughout the complex staining process. For the experimental study, all sections were stained in 1 continuous run. All microscopic analyses (see below) were done within 2 hours of completion of the staining protocol.

Microscopic and image analyses of human IHC samples. A renal pathologist without knowledge of the diagnosis performed the analysis. The slides were examined with an Olympus BX51 microscope with an epifluorescence attachment and photographed with an Olympus DP72 camera. The anti-CD14 Ab was examined with the TRITC filter, the anti-CD16 Ab with the FITC filter, and the anti-CD15 Ab with a light microscope. Several steps were followed for each glomerulus (Supplemental Figure 5). First, the glomerulus was centered and a photograph taken with the $\times 40$ objective using the FITC filter. Then, without movement of the slide, the TRITC filter was used for a second photograph, and finally, a third photograph of the IHC stain was taken with a light microscope. After reaction with PAS, the glomeruli were relocated and photographed with the light microscope. The microscopic TRITC, FITC, and IHC images were converted to 8-bit gray scale, and the IHC images were inverted so that all 3 images had comparable black backgrounds. A composite was made using Image J software (NIH). The composite and the PAS image were then opened in Adobe Photoshop (Adobe Systems), and the PAS image was manually aligned with the composite. The opacity of the PAS image was adjusted to identify cells with or without TRITC, FITC, and/or IHC reaction. $\mathrm{CD} 16^{+} \mathrm{CD} 14^{-}$cells were counted. For the IHC images, cells with or without CD16 and CD14 positivity were counted, and the photographs with the positive cells were labeled. For final illustration, the opacity of the PAS photograph was set at $45 \%$.

PB and flow cytometric analyses. PB was collected by retro-orbital bleeding of the mice. Complete blood cell counts (CBCs) were determined using the Forcyte Hematology System (Oxford Science). For flow cytometric analysis of PB, RBC were lysed with RBC Lysis Buffer according to the manufacturer's instructions (STEMCELL Technologies), and single-cell suspensions were obtained by passing cells through $40-\mu \mathrm{m}$ cell strainers. For immune cell analysis of kidneys, perfused kidneys were cut into 1- to 2- $\mathrm{mm}^{3}$ pieces and digested in DMEM (Thermo Fisher Scientific) containing $2 \mathrm{mg} / \mathrm{ml}$ collagenase type I (Worthington) and $2 \mathrm{mg} / \mathrm{ml}$ DNaseI (Worthington) for 30 minutes at $37^{\circ} \mathrm{C}$. Digested kidneys were passed through a $40-\mu \mathrm{m}$ cell strainer (BD Biosciences) to obtain single-cell suspensions, and cell numbers were determined with a hemocytometer (Neubauer chamber). Cells obtained from kidneys and PB were blocked with Abs against CD16 and CD32 (clone 2.4G2, Tonbo), followed by staining for cell-surface markers. In the PB, cells

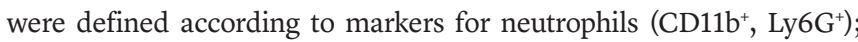
PMos $\left(\mathrm{CD}_{11 b^{+}}, \mathrm{Ly}_{6 \mathrm{G}}, \mathrm{CD} 15^{+}, \mathrm{Ly}_{6} \mathrm{C}^{\mathrm{lo}}, \mathrm{CD} 43^{\mathrm{hi}}\right)$; and classical mono-

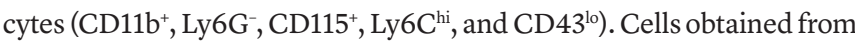
kidneys were defined according to markers for neutrophils $\left(\mathrm{CD} 11 \mathrm{~b}^{+}\right.$, Ly6G ${ }^{+}$) and PMos $\left(\mathrm{CD}_{11 \mathrm{~b}^{+}}\right.$, Ly6G $\mathrm{G}^{-}, \mathrm{F} 4 / 80^{\mathrm{lo}}$, and CD $\left.43^{\mathrm{hi}}\right)$. The following
Abs were used for flow cytometry: FITC-MHC-II (M5/114.15.2; eBioscience; 1:300 dilution); PE-CD115 (AFS98; eBioscience; 1:100 dilution); PE-F4/80 (BM8; eBioscience; 1:200 dilution); PE-Cy7-Ly-6G (1A8; BioLegend; 1:200 dilution); APC-CD43 (S7; BD Biosciences; 1:300 dilution); APC/Cy7-Ly6C (HK1.4; BioLegend; 1:200 dilution); BV421CD11b (M1/70; BioLegend; 1:200 dilution); and PerCP-Cy5.5-CD11c (N418; eBioscience; 1:100 dilution). The specificity of all Abs was controlled by the respective $\mathrm{Ab}$ isotypes. Samples were analyzed using a FACSCanto II (BD Biosciences) and FlowJo 7.6.5 software. Cell sorting was done using a FACSAria (BD Biosciences) or a Reflection (Sony Biotechnology) instrument. Absolute numbers of individual cell populations were calculated on the basis of total cell numbers from $\mathrm{CBC}$ (PB) and Neubauer chambers (kidneys) and the relative numbers of specific cell populations (defined by flow cytometry).

Adoptive transfer of Hoxb8-FL cells. Hoxb8-FL cells were generated as described previously (47). In brief, BM cells from Tnip $1^{+/+}$and Tnip1 $1^{-/-}$mice were infected with an MSCV-based vector expressing an estrogen-regulated form of Hoxb8 (ER-HBD-Hoxb8), and conditionally immortalized progenitor cells were expanded in the presence of FMS-like tyrosine kinase 3 ligand (FLT3L) and estrogen. Stably growing cells were labeled by retroviral transduction with murine stem cell virus-based (MSCV-based) vectors expressing EGFP or ametrine. Cells $\left(2.5 \times 10^{6}\right)$ expressing EGFP or ametrine (e.g., Hoxb8-FL cells from Tnip1 $1^{+/+}$[GFP] and Tnip1 $1^{-/}$mice [ametrine]) were mixed at a 1:1 ratio and injected i.v. into lethally irradiated mice along with $2 \times 10^{5}$ nonfractionated BM helper cells. Cells derived from Hoxb8-FL cells were identified on the basis of EGFP and ametrine expression.

In vivo Ab labeling. Tnip1 $1^{-/}$mice were injected i.v. with $3 \mu \mathrm{g}$ PE-conjugated anti-mouse CD45 Ab (clone 30-F11; eBioscience). Ten minutes later, mice were perfused with $20 \mathrm{ml}$ PBS as described above. Immune cells were isolated and analyzed by flow cytometry.

ELISA and proteinuria analysis. Total ANA Ig and anti-dsDNA Ig levels in the serum were determined with ELISA kits (Alpha Diagnostic International) according to the manufacturer's instructions. The protein concentration in urine was determined using Multistix 10 SG sticks (Siemens) and scored as 0 (negative), 1 ( $<30 \mathrm{mg} / \mathrm{dl}), 2$ ( $>30 \mathrm{mg} / \mathrm{dl}), 3$ (>100 mg/dl), and 4 (>300 mg/dl).

Immunoblot analysis. Cells were lysed in buffer containing $20 \mathrm{mM}$ HEPES and $\mathrm{KOH}$ (pH 7.5), $150 \mathrm{mM} \mathrm{NaCl}, 1 \mathrm{mM}$ EDTA, 0.5\% NP-40, $10 \%$ glycerol, $10 \mathrm{mM}$ pyrophosphate, supplemented with complete proteases inhibitors (Roche). Cell lysates were resolved by SDS-PAGE using precast gels (Bio-Rad) and transferred onto nitrocellulose membranes. Membranes were probed with Abs against ABIN1 (clone HPA037893; Atlas Antibodies) or GRB2 (catalog 3972; Cell Signaling Technology) and visualized using ECL (Thermo Fisher Scientific).

Transdermal GFR analysis. GFR measurements were performed in conscious mice as recently described $(68,69)$. In short, the transdermal GFR monitor (MediBeacon) was placed onto the side of the depilated back skin of briefly anesthetized mice using a double-sided adhesive patch and secured with additional adhesive tape around the body to minimize movement artifacts. The background fluorescence signal was recorded for 15 minutes, followed by i.v. injection of FITC-sinistrin (in PBS, $7 \mathrm{mg} / 100 \mathrm{~g}$ BW; Fresenius Kabi) and continuous fluorescence recording for 60 minutes. Recorded data were analyzed using MPD Lab software (Medibeacon), with the GFR ( $\mu \mathrm{l} / \mathrm{min} / 100 \mathrm{~g} \mathrm{BW})$ calculated on the basis of the decrease of fluorescence intensity of FITC-sinistrin over time using the 3-compartment model with linear correction. 
Isolation of kidney glomeruli. Mouse glomeruli were isolated by the sieving technique. Kidneys were perfused with $10 \mathrm{ml}$ PBS through the left ventricle after incision of the lateral left lobe of the liver. Kidneys were removed, decapsulated, and the cortex of each kidney separated from the medulla by macroscopic dissection. The cortex was gently strained through a 100- $\mu \mathrm{M}$ cell strainer in PBS containing 0.5\% FBS (PBS/FBS) using the plunger of a 3-ml syringe. The glomeruli-containing flow-through was collected and passed through a $40-\mu \mathrm{M}$ strainer, thereby retaining glomeruli. The $40-\mu \mathrm{M}$ cell strainer was then placed onto a $50-\mathrm{ml}$ tube, and the glomeruli were washed twice with $45 \mathrm{ml}$ PBS/FBS. The cell strainer was inverted onto a Petri dish, and the glomeruli were collected by flushing the cell strainer with PBS/FBS. The isolated glomeruli were examined by light microscopy to assess the purity.

Statistics. Data were analyzed with GraphPad Prism software and are expressed as the mean \pm SD or the mean \pm SEM, as indicated in the figure legends. Statistical significance between 2 groups was assessed with the Mann-Whitney $U$ test. Statistical significance between more than 2 groups was assessed by 1-way ANOVA with Tukey's multiple comparisons test. $P$ values of less than 0.05 were considered statistically significant.

Study approval. All mouse studies were carried out in accordance with protocols approved by the IACUC of St. Jude Children's Research Hospital. The retrospective analysis of human renal biopsy material was performed on deidentified material, and thus the study was exempt from the informed consent requirement. The protocol was approved by the Schulman Institutional Review Board, and the study was conducted according to Declaration of Helsinki principles.

\section{Author contributions}

JK performed experiments, generated figures, and analyzed data. VR performed experiments, analyzed data, generated figures, and participated in writing the manuscript. CG performed IF analyses and generated figures. JZ performed experiments and analyzed data. RW performed experiments. SKI performed experiments, analyzed data, and generated figures. HT analyzed data. PDW performed pathological analysis of the human samples. PV performed pathological analysis of the mouse models. HH conceived and supervised the project, performed experiments, analyzed data, and wrote the manuscript.

\section{Acknowledgments}

We gratefully acknowledge the staff of the Animal Resource Center and the Veterinary Pathology Core at St. Jude Children's Research Hospital. We also thank Susu Duan (St. Jude Children's Research Hospital) for help with Ab-labeling experiments. This work was supported by the Lupus Research Alliance and the American Lebanese Syrian Associated Charities (ALSAC).

Address correspondence to: Hans Häcker, Department of Infectious Diseases, St. Jude Children's Research Hospital, 262 Danny Thomas Place, Memphis, Tennessee 38105, USA. Phone: 901.595.4688; Email: hans.haecker@stjude.org.

SKI's present address is: Department of Biotechnology, All India Institute of Medical Sciences, New Delhi, India.
1. Maroz N, Segal MS. Lupus nephritis and end-stage kidney disease. Am JMed Sci. 2013;346(4):319-323.

2. Yu C, Gershwin ME, Chang C. Diagnostic criteria for systemic lupus erythematosus: a critical review. J Autoimmun. 2014;48-49:10-13.

3. Lau CM, et al. RNA-associated autoantigens activate B cells by combined B cell antigen receptor/Toll-like receptor 7 engagement. J Exp Med. 2005;202(9):1171-1177.

4. Barrat FJ, et al. Nucleic acids of mammalian origin can act as endogenous ligands for Toll-like receptors and may promote systemic lupus erythematosus. JExp Med. 2005;202(8):1131-1139.

5. Garcia-Romo GS, et al. Netting neutrophils are major inducers of type I IFN production in pediatric systemic lupus erythematosus. Sci Transl Med. 2011;3(73):73ra20.

6. Lande R, et al. Neutrophils activate plasmacytoid dendritic cells by releasing self-DNA-peptide complexes in systemic lupus erythematosus. $\mathrm{Sci}$ Transl Med. 2011;3(73):73ra19.

7. Häcker H, et al. CpG-DNA-specific activation of antigen-presenting cells requires stress kinase activity and is preceded by non-specific endocytosis and endosomal maturation. EMBOJ. 1998;17(21):6230-6240.

8. Pisitkun P, Deane JA, Difilippantonio MJ, Tarasenko T, Satterthwaite AB, Bolland S. Autoreactive B cell responses to RNA-related antigens due to TLR7 gene duplication. Science. 2006;312(5780):1669-1672.

9. Subramanian S, et al. A Tlr7 translocation accelerates systemic autoimmunity in murine lupus. Proc Natl Acad Sci U S A. 2006;103(26):9970-9975.

10. Ehlers M, Fukuyama H, McGaha TL, Ader- em A, Ravetch JV. TLR9/MyD88 signaling is required for class switching to pathogenic IgG2a and $2 \mathrm{~b}$ autoantibodies in SLE. J Exp Med. 2006;203(3):553-561.

11. Sadanaga A, et al. Protection against autoimmune nephritis in MyD88-deficient MRL/lpr mice. Arthritis Rheum. 2007;56(5):1618-1628.

12. Nickerson KM, et al. TLR9 regulates TLR7- and MyD88-dependent autoantibody production and disease in a murine model of lupus. JImmunol. 2010;184(4):1840-1848.

13. Santiago-Raber ML, et al. Critical role of TLR7 in the acceleration of systemic lupus erythematosus in TLR9-deficient mice. J Autoimmun. 2010;34(4):339-348.

14. Merrill JT, et al. Efficacy and safety of rituximab in moderately-to-severely active systemic lupus erythematosus: the randomized, double-blind, phase II/III systemic lupus erythematosus evaluation of rituximab trial. Arthritis Rheum. 2010;62(1):222-233.

15. Morris DL, et al. Genome-wide association meta-analysis in Chinese and European individuals identifies ten new loci associated with systemic lupus erythematosus. Nat Genet. 2016;48(8):940-946.

16. Mohan C, Putterman C. Genetics and pathogenesis of systemic lupus erythematosus and lupus nephritis. Nat Rev Nephrol. 2015;11(6):329-341.

17. Gateva V, et al. A large-scale replication study identifies TNIP1, PRDM1, JAZF1, UHRF1BP1 and IL10 as risk loci for systemic lupus erythematosus. Nat Genet. 2009;41(11):1228-1233.

18. Han JW, et al. Genome-wide association study in a Chinese Han population identifies nine new susceptibility loci for systemic lupus erythematosus. Nat Genet. 2009;41(11):1234-1237.

19. Caster DJ, et al. ABIN1 dysfunction as a genetic basis for lupus nephritis. J Am Soc Nephrol. 2013;24(11):1743-1754.

20. Adrianto I, et al. Association of two independent functional risk haplotypes in TNIP1 with systemic lupus erythematosus. Arthritis Rheum. 2012;64(11):3695-3705.

21. Heyninck K, et al. The zinc finger protein A2O inhibits TNF-induced NF-kappaB-dependent gene expression by interfering with an RIP- or TRAF2-mediated transactivation signal and directly binds to a novel NF-kappaB-inhibiting protein ABIN. J Cell Biol. 1999;145(7):1471-1482.

22. Zhou J, et al. A2O-binding inhibitor of NF- $\mathrm{KB}$ (ABIN1) controls Toll-like receptor-mediated CCAAT/enhancer-binding protein $\beta$ activation and protects from inflammatory disease. Proc Natl Acad Sci U S A. 2011;108(44):E998-1006.

23. Ippagunta $S K$, et al. Keratinocytes contribute intrinsically to psoriasis upon loss of Tnip1 function. Proc Natl Acad Sci U S A. 2016;113(41):E6162-E6171.

24. Santiago-Raber ML, Baudino L, Alvarez M, van Rooijen N, Nimmerjahn F, Izui S. TLR7/9mediated monocytosis and maturation of Gr-1(hi) inflammatory monocytes towards Gr-1(lo) resting monocytes implicated in murine lupus. JAutoimmun. 2011;37(3):171-179.

25. Cros J, et al. Human CD14dim monocytes patrol and sense nucleic acids and viruses via TLR7 and TLR8 receptors. Immunity. 2010;33(3):375-386.

26. Carlin LM, et al. Nr4a1-dependent Ly6C(low) monocytes monitor endothelial cells and orches- 
trate their disposal. Cell. 2013;153(2):362-375.

27. Finsterbusch $\mathrm{M}$, et al. Patrolling monocytes promote intravascular neutrophil activation and glomerular injury in the acutely inflamed glomerulus. Proc Natl Acad Sci U S A. 2016;113(35):E5172-E5181.

28. Imhof BA, et al. CCN1/CYR61-mediated meticulous patrolling by Ly6Clow monocytes fuels vascular inflammation. Proc Natl Acad Sci U S A. 2016;113(33):E4847-E4856.

29. Mukherjee R, Kanti Barman P, Kumar Thatoi P, Tripathy R, Kumar Das B, Ravindran B. Non-classical monocytes display inflammatory features: validation in sepsis and systemic lupus erythematosus. Sci Rep. 2015;5:13886.

30. Yoshimoto S, et al. Elevated levels of fractalkine expression and accumulation of $\mathrm{CD}_{16}{ }^{+}$monocytes in glomeruli of active lupus nephritis. Am J Kidney Dis. 2007;50(1):47-58.

31. Weening JJ, et al. The classification of glomerulonephritis in systemic lupus erythematosus revisited. J Am Soc Nephrol. 2004;15(2):241-250.

32. Takeuchi E, Iizuka M, Tamura M, Takeuchi Y. Convenient evaluation of magnitude of glomerulonephritis in BXSB/Mp lupus mice. JClin Cell Immunol. 2014;5(2):http://dx.doi. org/10.4172/2155-9899.1000209.

33. Häcker H, Vabulas RM, Takeuchi O, Hoshino $\mathrm{K}$, Akira S, Wagner H. Immune cell activation by bacterial CpG-DNA through myeloid differentiation marker 88 and tumor necrosis factor receptor-associated factor (TRAF) 6. J Exp Med. 2000;192(4):595-600.

34. Häcker H, et al. Specificity in Toll-like receptor signalling through distinct effector functions of TRAF3 and TRAF6. Nature. 2006;439(7073):204-207.

35. Wu YW, Tang W, Zuo JP. Toll-like receptors: potential targets for lupus treatment. Acta Pharmacol Sin. 2015;36(12):1395-1407.

36. Liu Y, Seto NL, Carmona-Rivera C, Kaplan MJ. Accelerated model of lupus autoimmunity and vasculopathy driven by toll-like receptor $7 / 9$ imbalance. Lupus Sci Med. 2018;5(1):e000259.

37. Mombaerts P, Iacomini J, Johnson RS, Herrup K, Tonegawa S, Papaioannou VE. RAG-1-deficient mice have no mature B and T lymphocytes. Cell. 1992;68(5):869-877.

38. Chan OT, Hannum LG, Haberman AM, Madaio MP, Shlomchik MJ. A novel mouse with B cells but lacking serum antibody reveals an antibody-independent role for $\mathrm{B}$ cells in murine lupus. J Exp Med. 1999;189(10):1639-1648.

39. Noris M, Remuzzi G. Overview of complement activation and regulation. Semin Nephrol. 2013;33(6):479-492.

40. Davidson A. What is damaging the kidney in lupus nephritis? Nat Rev Rheumatol. 2016;12(3):143-153.
41. Merad M, Sathe P, Helft J, Miller J, Mortha A. The dendritic cell lineage: ontogeny and function of dendritic cells and their subsets in the steady state and the inflamed setting. Annu Rev Immunol. 2013;31:563-604.

42. Sung SJ, et al. Dependence of glomerulonephritis induction on novel intraglomerular alternatively activated bone marrow-derived macrophages and Mac-1 and PD-L1 in lupus-prone NZM2328 mice. JImmunol. 2017;198(7):2589-2601.

43. Hanna RN, et al. The transcription factor NR4A1 (Nur77) controls bone marrow differentiation and the survival of Ly6C- monocytes. Nat Immunol. 2011;12(8):778-785.

44. Watanabe-Fukunaga R, Brannan CI, Copeland NG, Jenkins NA, Nagata S. Lymphoproliferation disorder in mice explained by defects in Fas antigen that mediates apoptosis. Nature. 1992;356(6367):314-317.

45. Clausen BE, Burkhardt C, Reith W, Renkawitz $\mathrm{R}$, Förster I. Conditional gene targeting in macrophages and granulocytes using LysMcre mice. Transgenic Res. 1999;8(4):265-277.

46. Lamagna C, Scapini P, van Ziffle JA, DeFranco AL, Lowell CA. Hyperactivated MyD88 signaling in dendritic cells, through specific deletion of Lyn kinase, causes severe autoimmunity and inflammation. Proc Natl Acad Sci U S A 2013;110(35):E3311-E3320.

47. Redecke V, et al. Hematopoietic progenitor cell lines with myeloid and lymphoid potential. Nat Methods. 2013;10(8):795-803.

48. Auffray C, et al. Monitoring of blood vessels and tissues by a population of monocytes with patrolling behavior. Science. 2007;317(5838):666-670

49. Palumbo-Zerr K, et al. Orphan nuclear receptor NR4A1 regulates transforming growth factor- $\beta$ sig naling and fibrosis. Nat Med. 2015;21(2):150-158.

50. Inoue A, et al. Antagonist of fractalkine (CX3CL1) delays the initiation and ameliorates the progression of lupus nephritis in MRL/lpr mice. Arthritis Rheum. 2005;52(5):1522-1533.

51. Miles S, Isenberg D. A review of serological abnormalities in relatives of SLE patients. Lupus. 1993;2(3):145-150.

52. Stamatiades EG, et al. Immune Monitoring of Trans-endothelial Transport by Kidney-Resident Macrophages. Cell. 2016;166(4):991-1003.

53. Chalmers SA, Wen J, Shum J, Doerner J, Herlitz L, Putterman C. CSF-1R inhibition attenuates renal and neuropsychiatric disease in murine lupus. Clin Immunol. 2017;185:100-108.

54 . Westbrook L, et al. Genetic susceptibility and loss of Nr4a1 enhances macrophage-mediated renal injury in CKD. J Am Soc Nephrol. 2014;25(11):2499-2510.

55. Liebmann M, et al. Nur77 serves as a molecular brake of the metabolic switch during T cell acti- vation to restrict autoimmunity. Proc Natl Acad Sci U S A. 2018;115(34):E8017-E8026.

56. Courtney PA, Crockard AD, Williamson K, Irvine AE, Kennedy RJ, Bell AL. Increased apoptotic peripheral blood neutrophils in systemic lupus erythematosus: relations with disease activity, antibodies to double stranded DNA, and neutropenia. Ann Rheum Dis. 1999;58(5):309-314.

57. Smith CK, Kaplan MJ. The role of neutrophils in the pathogenesis of systemic lupus erythematosus. Curr Opin Rheumatol. 2015;27(5):448-453.

58. Knight JS, et al. Peptidylarginine deiminase inhibition disrupts NET formation and protects against kidney, skin and vascular disease in lupus-prone MRL/lpr mice. Ann Rheum Dis. 2015;74(12):2199-2206.

59. Knight JS, et al. Peptidylarginine deiminase inhibition is immunomodulatory and vasculoprotective in murine lupus. JClin Invest. 2013;123(7):2981-2993.

60. Clynes R, Dumitru C, Ravetch JV. Uncoupling of immune complex formation and kidney damage in autoimmune glomerulonephritis. Science. 1998;279(5353):1052-1054

61. Wessels MR, Butko P, Ma M, Warren HB, Lage AL, Carroll MC. Studies of group B streptococcal infection in mice deficient in complement component $\mathrm{C} 3$ or $\mathrm{C} 4$ demonstrate an essential role for complement in both innate and acquired immunity. Proc Natl Acad Sci U S A. 1995;92(25):11490-11494.

62. Adachi $\mathrm{O}$, et al. Targeted disruption of the MyD88 gene results in loss of IL-1- and IL-18mediated function. Immunity. 1998;9(1):143-150.

63. Hemmi H, et al. Small anti-viral compounds activate immune cells via the TLR7 MyD88dependent signaling pathway. Nat Immunol. 2002;3(2):196-200.

64. Hemmi H, et al. A Toll-like receptor recognizes bacterial DNA. Nature. 2000;408(6813):740-745

65. Lee SL, Wesselschmidt RL, Linette GP, Kanagawa O, Russell JH, Milbrandt J. Unimpaired thymic and peripheral $\mathrm{T}$ cell death in mice lacking the nuclear receptor NGFI-B (Nur77). Science. 1995;269(5223):532-535.

66 . Weening JJ, et al. The classification of glomerulonephritis in systemic lupus erythematosus revisited. Kidney Int. 2004;65(2):521-530.

67. Zhang W, et al. Fully automated 5-plex fluorescent immunohistochemistry with tyramide signal amplification and same species antibodies. Lab Invest. 2017;97(7):873-885.

68. Schreiber A, et al. Transcutaneous measurement of renal function in conscious mice. Am JPhysio Renal Physiol. 2012;303(5):F783-F788.

69. Friedemann J, et al. Improved kinetic model for the transcutaneous measurement of glomerular filtration rate in experimental animals. Kidney Int. 2016;90(6):1377-1385. 